\title{
A New Class of 'Tethered' Ruthenium (II) Catalyst for Asymmetric Transfer Hydrogenation Reactions.
}

\author{
Jérôme Hannedouche, ${ }^{a}$ Guy Clarkson ${ }^{\mathrm{b}}$ and Martin Wills ${ }^{\mathrm{a} *}$ \\ ${ }^{a}$ Asymmetric Catalysis Group, ${ }^{b} X$-ray Crystallography Unit, \\ Department of Chemistry, University of Warwick, Coventry, CV4 7AL. \\ (Supporting Information)
}

\section{Contents}

A) General: $\quad$ S3

$\begin{array}{ll}\text { B) Materials: } & \text { S3 }\end{array}$

C) Synthesis of $(R, R)$-(N-2-cyclohexa-1,4-dienyl-ethanesulfonyl)-1,2-diphenylethylene-diamine $6 \quad \mathrm{~S} 3$

1) Synthesis of 2-phenyl-ethanesulfonic acid A $\quad S 3$

2) Synthesis of 2-cyclohexa-1,4-dienyl-ethanesulfonyl chloride B $\quad S 4$

3) Synthesis of $(R, R)$-(N-2-cyclohexa-1,4-dienyl-ethanesulfonyl)-1,2-diphenylethylene-diamine $6 \quad \mathrm{~S} 4$

D) Synthesis of ruthenium (II) dimer $4 \quad S 4$

E) Synthesis of ruthenium(II) catalyst $2 \quad$ S4

F) Experimental procedure for the asymmetric transfer hydrogenation catalysed by ruthenium (II) dimer $\mathbf{4}$ and 2S5

G) HPLC , GC analysis and optical rotation data of the reduction products $\quad$ S5

H) Synthesis of $(1 R, 2 S)$-(N-3-cyclohexa-1,4-dienyl-propyl)-norephedrine $5 \quad$ S5

1) Synthesis of 3-cyclohexa-1,4-dienyl-propan-1-ol C $\quad$ S5

2) Synthesis of 3-cyclohexa-1,4-dienyl-propionaldehyde D $\quad$ S6

3) Synthesis of $(1 R, 2 S)$-(N-3-cyclohexa-1,4-dienyl-propyl)-norephedrine $5 \quad$ S6

I) Synthesis of the chloro-bridged $\eta^{6}$-arene ruthenium (II) dimer $\mathbf{3} \quad \mathrm{S} 6$

J) Experimental procedure for the asymmetric transfer hydrogenation catalysed by ruthenium (II) dimer $3 \quad$ S7

$\begin{array}{ll}\text { K) HPLC , GC analysis and optical rotation data of the reduction products } & \text { S7 }\end{array}$

L) ${ }^{1} \mathrm{H}$ and ${ }^{13} \mathrm{C}$ NMR spectra $\quad$ S8

1) Spectra of 2-phenyl-ethanesulfonic acid A $\quad$ S8

2) Spectra of 2-cyclohexa-1,4-dienyl-ethanesulfonyl chloride $\mathbf{B} \quad S 9$

3) Spectra of $(R, R)$-(N-2-cyclohexa-1,4-dienyl-ethanesulfonyl)-1,2-diphenylethylene-diamine $6 \quad \mathrm{~S} 10$

4) Spectra of Ruthenium (II) dimer $4 \quad$ S11 
5) Spectra of 3-cyclohexa-1,4-dienyl-propan-1-ol C

$\mathrm{S} 12$

6) Spectra of 3-cyclohexa-1,4-dienyl-propionaldehyde $\mathbf{D}$

$\mathrm{S} 13$

7) Spectra of $(1 R, 2 S)$-(N-3-cyclohexa-1,4-dienyl-propyl)-norephedrine $5 \quad \mathrm{~S} 14$

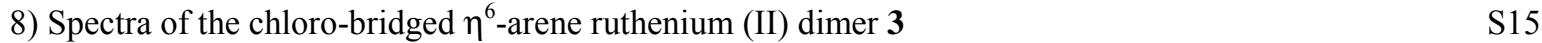

M) Single X-ray data analysis data of $2 \quad$ S16

1) ORTEP plot of the molecular structure $\quad$ S16

2) Experimental Details $\quad$ S16

3) Table 1. Atomic coordinates and equivalent isotropic displacement parameters $\quad$ S19

4) Table 2. Bond lengths and angles $\quad$ S20

5) Table 3. Anisotropic displacement parameters $\quad$ S24

6) Table 4. Hydrogen coordinates and isotropic displacement parameters $\quad$ S25

$\begin{array}{lr}\text { N) References } & \text { S26 }\end{array}$ 


\section{A) General:}

All reactions, unless otherwise stated, were run under an atmosphere of nitrogen in flame or oven dried glassware (round bottomed flasks or Schlenk tubes). Room temperature refers to ambient room temperature $\left(20-22{ }^{\circ} \mathrm{C}\right), 0{ }^{\circ} \mathrm{C}$ refers to an ice slush bath and $-78^{\circ} \mathrm{C}$ refer to a dry-ice acetone bath. Heated experiments were conducted using thermostatically controlled oil baths. Reactions were monitored by Thin Layer Chromatography (TLC) using aluminium backed silica gel 60 ( $\left.\mathrm{F}_{254}\right)$ plates, visualised using $\mathrm{UV}_{254 \mathrm{mn}}$ and PMA, potassium permanganate or ninhydrin dips as appropriate. Flash column chromatography was carried out routinely using $60 \AA$ silica gel (Merck). NMR spectra were recorded on Bruker DPX-300 (300Mhz) or DXP 400 (400 Mhz) spectrometer. Chemical shifts are reported in $\delta$ units, parts per million downfield from $\left(\mathrm{CH}_{3}\right)_{4} \mathrm{Si}$. Coupling constants $(\mathrm{J})$ are measured in hertz. IR spectra were recorded on a Nicolet Model Avatar 320 FTIR instrument. Mass spectra were recorded on a 7070 E VG mass spectrometer. Determination of enantiomeric excesses by HPLC analysis (using a Merck-Hitachi L-6200 A intelligent pump, Merck-Hitachi L-4000 UV detector, Axxiom chromatography data system and controller model 727 and a Daicel Chiralcel OD 4.6 × $250 \mathrm{~mm}$ column) or GC analysis (Hewlett Packard 5890A gas chromatography, Hewlett Packard 3396A integrator and Supelco BETA Dex ${ }^{\mathrm{TM}} 120$ fused silica capillary column $30 \mathrm{~m}$ x $0.25 \mathrm{~mm}$ x $0.25 \mu \mathrm{m}$ film thickness) as stated. Elemental analyses were performed using the Exeter Analytical Model CE440. Melting points were recorded on a Stuart Scientific SMP1 instrument and are uncorrected. Optical rotations were measured on a Perkin-Elmer 241 polarimeter.

\section{B) Materials:}

Commercially available reagents were used without purification: 3-phenyl-propan-1-ol (Aldrich), oxalylchloride (Aldrich), dimethylsulfoxide (Lancaster), L-(-)-norephedrine (Fluka), benzenethanethiol (Aldrich), (R,R)-1,2-diphenylethylenediamine (Fluka), hydrate ruthenium (III) trichloride (Apollo), peracetic acid (36-40\% solution in acetic acid) (Aldrich), triethylamine (99\%) (Lancaster), thionyl chloride (Aldrich), hydrochloric acid (2M solution in diethyl ether) (Aldrich), sodium (stick in paraffin) (BDH) and ammonia (BOC). All anhydrous solvents were used as supplied by Romil in HyDry ${ }^{\mathrm{TM}}$, except dimethylformamide and propan-2-ol, which were supplied by Aldrich. Formic acid: triethylamine mixture (5:2 molar) is a commercially available azeotrope (Fluka).

\section{C) Synthesis of $(R, R)$-(N-2-cyclohexa-1,4-dienyl-ethanesulfonyl)-1,2-diphenylethylene-diamine 6}

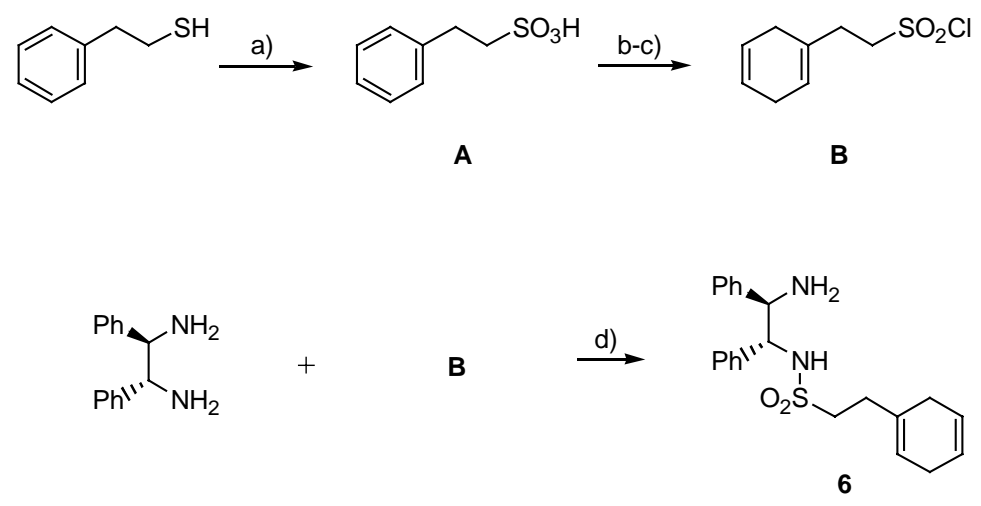

a) $\mathrm{CH}_{3} \mathrm{CO}_{3} \mathrm{H}\left(36-40 \%\right.$ in acetic acid) (3 eq), DCM, rt, $2 \mathrm{~h}$; b) $\mathrm{Na}, \mathrm{NH}_{3}, \mathrm{EtOH},-78^{\circ} \mathrm{C}$, $3 \mathrm{~h} ; \mathrm{c}) \mathrm{SOCl}_{2}$ (20 eq), DMF (2 eq), DCM, $\left.35^{\circ} \mathrm{C}, 16 \mathrm{~h} ; \mathrm{d}\right) \mathrm{Et}_{3} \mathrm{~N}(2 \mathrm{eq}), \mathrm{DCM}, \mathrm{rt}, \mathrm{o} / \mathrm{h}$.

\section{1) Synthesis of 2-phenyl-ethanesulfonic acid $\mathrm{A}^{\square}$}

To a stirred solution of benzenethanethiol $(9 \mathrm{~g}, 65 \mathrm{mmol})$ in dichloromethane $(285 \mathrm{~mL})$ was dropwise added peracetic acid ( 36.5 $\mathrm{mL}, 195 \mathrm{mmol})$ at $0^{\circ} \mathrm{C}$. The reaction mixture was warmed to room temperature and stirred for $2 \mathrm{~h}$. Then the solution was concentrated under high-reduced pressure at $40^{\circ} \mathrm{C}$ to afford a viscous yellow oil $(12 \mathrm{~g}, 100 \%$ yield $)$ that turns red after few hours. IR $v_{\max }\left(\mathrm{cm}^{-1}\right) 3368,3026,1682,1105$ and $1016 ;{ }^{1} \mathrm{H} \mathrm{NMR}\left(400 \mathrm{MHz}, \mathrm{CDCl}_{3}\right) \delta_{\mathrm{H}}(\mathrm{ppm}) 11.00(\mathrm{br} \mathrm{s}, 2 \mathrm{H}), 7.27-7.14(\mathrm{~m}, 5 \mathrm{H}), 3.33-$ 
$3.11(\mathrm{~m}, 2 \mathrm{H})$ and 3.10-3.07 (m, 2H); ${ }^{13} \mathrm{C}$ NMR (100 MHz, $\left.\mathrm{CDCl}_{3}\right) \delta_{\mathrm{C}}(\mathrm{ppm}) 137.9,129.2,128.8,127.3,53.3$ and 30.3 ; MS (EI) m/z 186 ([M] $\left.]^{+}, 12\right), 169$ (5), 104 (100), 91 (26) and 77 (25\%); HRMS (EI) m/z calculated for $\mathrm{C}_{8} \mathrm{H}_{10} \mathrm{O}_{3} \mathrm{~S}$ [M] $]^{+}$186.0350. Found 186.0358 .

\section{2) Synthesis of 2-cyclohexa-1,4-dienyl-ethanesulfonyl chloride B}

To a refluxing solution of ammonia $2(177 \mathrm{~mL})$ was slowly added a solution of 2-phenyl-ethanesulfonic acid $\mathbf{A}(5.0 \mathrm{~g}, 26 \mathrm{mmol})$ in ethanol $(9 \mathrm{~mL})$ at $-78^{\circ} \mathrm{C}$ while stirring. Small pieces of cleaned sodium were added to the reaction mixture until the blue coloured persisted. During the addition of sodium, a small amount of ethanol was several times added to facilitate stirring. After the addition of sodium over the course of $3 \mathrm{~h}$, the ammonia was allowed to evaporate at room temperature overnight. The solution was filtered off and the residue was washed several times with ethanol $(10 \mathrm{~mL})$ to afford a slight orange solid. To a stirred suspension of the solid in anhydrous dichloromethane $(350 \mathrm{~mL})$ was added thionyl chloride $(37 \mathrm{~mL}, 520 \mathrm{mmol})$, followed by anhydrous dimethylformamide $(4 \mathrm{~mL}, 52 \mathrm{mmol})$ at $0^{\circ} \mathrm{C}$. The mixture was heated at $35^{\circ} \mathrm{C}$ for $16 \mathrm{~h}$ and filtered off. The filtrate was concentrated under reduced pressure to give an orange oil which was purified by flash chromatography $(10 \rightarrow 20 \% \mathrm{v} / \mathrm{v}$ ethyl acetate: hexane) to afford 2-cyclohexa-1,4-dienyl-ethanesulfonyl chloride $\mathbf{B}$ as a colourless oil (3.9 g, $73 \%$ yield). IR $v_{\max }\left(\mathrm{cm}^{-1}\right)$ 3029, 2921, 2854, 2820, 1367 and 1164; ${ }^{1} \mathrm{H} \mathrm{NMR}\left(400 \mathrm{MHz}, \mathrm{CDCl}_{3}\right) \delta_{\mathrm{H}}(\mathrm{ppm}) 5.71(\mathrm{br} \mathrm{s}, 2 \mathrm{H}), 5.58(\mathrm{br} \mathrm{s}, 1 \mathrm{H}), 3.78-3.74(\mathrm{~m}, 2 \mathrm{H})$ and 2.74-2.61 (m, 6H); $\left.{ }^{13} \mathrm{C} \mathrm{NMR} \mathrm{(100} \mathrm{MHz,} \mathrm{CDCl}_{3}\right) \delta_{\mathrm{C}}(\mathrm{ppm}) 129.8,124.5,123.7,122.8,64.2,32.1,29.2$ and 27.10; MS (EI) $\mathrm{m} / \mathrm{z} 208\left(\left[\mathrm{M}\left({ }^{37} \mathrm{Cl}\right)\right]^{+}, 15\right), 206\left(\left[\mathrm{M}\left({ }^{35} \mathrm{Cl}\right)\right]^{+}, 42\right), 171(8 \%), 153(14 \%), 105(100 \%), 91(99 \%)$ and $79(83 \%)$; Anal. Calculated for $\mathrm{C}_{8} \mathrm{H}_{11} \mathrm{ClO}_{2} \mathrm{~S}$ : C, 46.49; H, 5.36; Cl; 17.15; S, 15.51. Found: C, 46.22; H, 5.22; Cl; 17.12; S, 15.32 .

\section{3) Synthesis of $(R, R)$-(N-2-cyclohexa-1,4-dienyl-ethanesulfonyl)-1,2-diphenylethylene-diamine $6^{\text {— }}$}

To a stirred solution of $(R, R)$-1,2-diphenylethylene-diamine $(1.00 \mathrm{~g}, 4.7 \mathrm{mmol})$ in anhydrous dichloromethane $(20 \mathrm{~mL})$ was added triethylamine $(1.3 \mathrm{~mL}, 9.4 \mathrm{mmol})$, followed by 2-cyclohexa-1,4-dienyl-ethanesulfonyl chloride $\mathbf{B}(0.97 \mathrm{~g}, 4.7 \mathrm{mmol})$ at room temperature. The reaction mixture was stirred overnight at room temperature and concentrated to give a yellow oil which was purified by flash chromatography $(10 \rightarrow 80 \% \mathrm{v} / \mathrm{v}$ ethyl acetate: hexane) to afford a white solid (1.08 g, 60\% yield). mp 123-124 ${ }^{\circ} \mathrm{C} ;[\alpha]_{\mathrm{D}}{ }^{20}+20.4\left(c 1.00, \mathrm{CHCl}_{3}\right) ; \mathrm{IR} v_{\max }\left(\mathrm{cm}^{-1}\right) 3343,3290,3143,3025,2870,2816,1599,1451,1313$ and $1129 ;{ }^{1} \mathrm{H} \mathrm{NMR}(400$ $\left.\mathrm{MHz}, \mathrm{CDCl}_{3}\right) \delta_{\mathrm{H}}(\mathrm{ppm}) 7.32-7.24(\mathrm{~m}, 10 \mathrm{H}) ; 5.86($ br s, $1 \mathrm{H}), 5.64(\mathrm{br} \mathrm{s}, 2 \mathrm{H}), 5.18(\mathrm{br} \mathrm{s}, 1 \mathrm{H}), 4.56(\mathrm{~d}, J=5.5 \mathrm{~Hz}, 1 \mathrm{H}), 4.24(\mathrm{~d}, J=$ $5.5 \mathrm{~Hz}, 1 \mathrm{H}), 2.62-2.58(\mathrm{~m}, 2 \mathrm{H}), 2.55-2.47(\mathrm{~m}, 1 \mathrm{H}), 2.45-2.37(\mathrm{~m}, 1 \mathrm{H}), 2.32-2.28(\mathrm{~m}, 2 \mathrm{H}), 2.17-2.13(\mathrm{~m}, 2 \mathrm{H})$ and $1.53(\mathrm{br} \mathrm{s}, 4 \mathrm{H})$; ${ }^{13} \mathrm{C} \mathrm{NMR}\left(100 \mathrm{MHz}, \mathrm{CDCl}_{3}\right) \delta_{\mathrm{C}}(\mathrm{ppm}) 142.3,140.3,131.6,129.1,129.0,128.3,128.2,127.4,127.2,124.4,124.1$ and 120.5; MS (EI) $\mathrm{m} / \mathrm{z} 381\left([\mathrm{M}-\mathrm{H}]^{+}, 6\right), 211(4), 196(7), 106$ (100) and $79(26 \%)$; Anal. Calculated for $\mathrm{C}_{22} \mathrm{H}_{26} \mathrm{~N}_{2} \mathrm{O}_{2} \mathrm{~S}: \mathrm{C}, 69.08 ; \mathrm{H}, 6.85 ; \mathrm{N}$; 7.32. Found: C, 68.79; H, 6.80; N, 7.14.

\section{D) Synthesis of ruthenium (II) dimer $4^{\mathbb{1}}$}

To a stirred solution of $(R, R)$-(N-2-cyclohexa-1,4-dienyl-ethanesulfonyl)-1,2-diphenylethylene-diamine 6 (1.08 g, $2.8 \mathrm{mmol})$ in anhydrous dichloromethane $(20 \mathrm{~mL})$ was added a $2 \mathrm{M}$ solution of hydrochloric acid in diethyl ether $(4.2 \mathrm{~mL}, 8.5 \mathrm{mmol})$ at $0{ }^{\circ} \mathrm{C}$. The reaction mixture was stirred 5 minutes at room temperature and concentrated under reduced pressure to give a white residue. To a suspension of the residue in ethanol $(50 \mathrm{~mL})$ was added hydrate ruthenium (III) trichloride $(0.46 \mathrm{~g}, 1.8 \mathrm{mmol})$. The reaction mixture was stirred at reflux for $21 \mathrm{~h}$ and concentrated until dryness. The residue was washed several times with anhydrous dichloromethane to afford a khaki solid $\left(0.70 \mathrm{~g}, 66 \%\right.$ yield). ). $\mathrm{mp} 240-241{ }^{\circ} \mathrm{C}$ (dec.); IR $v_{\max }\left(\mathrm{cm}^{-1}\right) 3057,2916,2361,2339$, 1494, 1455, 1318 and $1143 ;{ }^{1} \mathrm{H}$ NMR $(300 \mathrm{MHz}, \mathrm{DMSO}) \delta_{\mathrm{H}}(\mathrm{ppm}) 8.78$ (br s, 3H), $8.53(\mathrm{~d}, J=9.6 \mathrm{~Hz}, 1 \mathrm{H}), 7.29-7.15(\mathrm{~m}, 10 \mathrm{H})$, 5.99-5.94 (m, 2H), 5.79-5.73 (m, 2H), $5.44(\mathrm{~d}, J=5.8 \mathrm{~Hz}, 1 \mathrm{H}), 4.74(\mathrm{dd}, J=9,9 \mathrm{~Hz}, 1 \mathrm{H}), 4.53(\mathrm{br}, 1 \mathrm{H}), 3.33-3.24(\mathrm{~m}$, $1 \mathrm{H}), 2.83-2.71(\mathrm{~m}, 2 \mathrm{H}), 2.62-2.54\left(\mathrm{~m}, 1 \mathrm{H}\right.$, slight overlap with DMSO peak); ${ }^{13} \mathrm{C}$ NMR $(100 \mathrm{MHz}, \mathrm{DMSO}) \delta_{\mathrm{C}}(\mathrm{ppm}) 138.1,134.4$, $128.7,128.4,127.9,127.8,102.9,88.0,86.1,85.9,84.2,61.0,58.2,51.5$ and 26.7; HRMS (LSIMS) m/z calculated for

${ }^{102} \mathrm{RuC}_{22} \mathrm{H}_{24} \mathrm{~N}_{2} \mathrm{O}_{2} \mathrm{SCl}$ 517.0290. Found 517.0299. This result corresponds to direct conversion to compound 3 in the mass spectrometer; the dimer could not observed by LSIMS or ESIMS due to this facile rearrangement. A detailed ESIMS analysis by the EPSRC MS service identified ions corresponding to $\mathrm{M}-3 \mathrm{HCl}-\mathrm{Cl}$ for the dimer.

\section{E) Synthesis of ruthenium(II) catalyst $2^{\text {B }}$}

To a suspension of ruthenium (II) dimer $4(0.200 \mathrm{~g}, 0.17 \mathrm{mmol})$ in propan-2-ol $(10 \mathrm{~mL})$ was added triethylamine $(0.19 \mathrm{~mL}, 1.4$ $\mathrm{mmol})$ at room temperature. The reaction mixture was refluxed for $1 \mathrm{~h}$ and concentrated under reduced pressure to give a brown residue, which was washed several times with anhydrous dichloromethane and dried in vacuo to afford a dark brown powder (0.101 g, 57\% yield). mp 258-260 ${ }^{\circ} \mathrm{C}$ (dec.); HRMS (LSIMS) m/z calculated for $\mathrm{RuC}_{22} \mathrm{H}_{24} \mathrm{~N}_{2} \mathrm{O}_{2} \mathrm{SCl}[\mathrm{M}+\mathrm{H}]^{+}$517.0290. Found 517.0289. Recrystallisation from acetonitrile afford orange crystals which the molecular structure was determined by single crystal X-ray analysis (see section $\mathrm{H}$ ). 
F) General experimental procedure for the asymmetric transfer hydrogenation catalysed by ruthenium (II) dimer 4 and 2

A solution of ruthenium (II) dimer $2\left(0.010 \mathrm{~g}, 8.3 \times 10^{-3} \mathrm{mmol}\right)$ in formic acid/ triethylamine $(5: 2)$ mixture $(1.5 \mathrm{~mL}) \mathrm{was}$ stirred at $28^{\circ} \mathrm{C}$ for 10 minutes then ketone $(3.3 \mathrm{mmol})$ was added. The reaction mixture was stirred at $28^{\circ} \mathrm{C}$ until completion and filtered through a pad of silica gel. The pad was washed with a $40 \%$ solution of ethyl acetate in hexane $(100 \mathrm{~mL})$. The filtrate was concentrated under reduced pressure to afford the reduction product. The same procedure was employed for the reduction using $\mathbf{2}$.

G) HPLC, GC analysis and optical rotation data of the reduction products

(R)-1-phenylethanol: $[\alpha]_{\mathrm{D}}{ }^{27}+54.9\left(c 1.00, \mathrm{CHCl}_{3}\right)\left(\right.$ lit. ${ }^{\mathrm{H}}[\alpha]_{\mathrm{D}}{ }^{23}+48.6\left(c 1.0, \mathrm{CH}_{2} \mathrm{Cl}_{2}\right), 96 \%$ e.e. $\left.(R)\right), 96 \%$ e.e. $(R)$ by $\mathrm{GC}$ (Chiracel $\beta$-DEX $120, \mathrm{~T}=115^{\circ} \mathrm{C}, \mathrm{P}=11 \mathrm{psi}, R$ isomer $8.7 \mathrm{~min}, S$ isomer $9.2 \mathrm{~min}$ ).

(R)-1-indanol: $[\alpha]_{\mathrm{D}}{ }^{27}-30.5\left(c 1.00, \mathrm{CHCl}_{3}\right)\left(\mathrm{lit}_{[}[\alpha]_{\mathrm{D}}{ }^{23}+30.9\left(c 1.99, \mathrm{CHCl}_{3}\right), 92 \%\right.$ e.e. $\left.(S)\right), 84 \%$ e.e. $(R)$ by HPLC (chiralcel OD column, propan-2-ol:hexane $=2: 98(1 \mathrm{~mL} / \mathrm{min}), S$ isomer $13.8 \mathrm{~min}, R$ isomer $15.8 \mathrm{~min})$.

(R)-1-tetralol: $[\alpha]_{\mathrm{D}}{ }^{27}-32.3\left(c 1.00, \mathrm{CHCl}_{3}\right)\left({ }_{\text {lit. }}{ }^{[}[\alpha]_{\mathrm{D}}+34.4\left(c 1.01, \mathrm{CHCl}_{3}\right), 98 \%\right.$ e.e. $\left.(S)\right), 98 \%$ e.e. $(R)$ by HPLC (chiralcel OD column, propan-2-ol:hexane $=2: 98(1 \mathrm{~mL} / \mathrm{min}), S$ isomer $10.7 \mathrm{~min}, R$ isomer $11.7 \mathrm{~min})$.

(R)-1-(2'-naphthyl)ethanol: $[\alpha]_{\mathrm{D}}{ }^{27}+45.4\left(c 1.00, \mathrm{CHCl}_{3}\right)\left(\right.$ lit. $^{8}[\alpha]_{\mathrm{D}}-34.3(c 1.10$, EtOH $\left.)\right), 86 \%$ e.e. $\left.(S)\right)$, $90 \%$ e.e. $(R)$ by HPLC (chiralcel OD column, ethanol:hexane $=5: 95(1 \mathrm{~mL} / \mathrm{min}), S$ isomer $14.7 \mathrm{~min}, R$ isomer $16.4 \mathrm{~min})$.

(R)-1-(p-chlorophenyl)ethanol: $[\alpha]_{\mathrm{D}}{ }^{27}+44.2\left(c 1.00\right.$, ether) (lit. $[\alpha]_{\mathrm{D}}{ }^{21}+46.1(c 0.9-1.1$, ether), $91 \%$ e.e. $(R))$, $92 \%$ e.e. $(R)$ by $\mathrm{GC}$ (Chiracel $\beta$-DEX 120, T $=140^{\circ} \mathrm{C}, \mathrm{P}=11 \mathrm{psi}, R$ isomer $11.1 \mathrm{~min}, S$ isomer $11.8 \mathrm{~min}$ ).

(R)-1-(p-methoxyphenyl)ethanol: $[\alpha]_{\mathrm{D}}{ }^{27}+32.3\left(c 1.00, \mathrm{CHCl}_{3}\right)\left(\right.$ lit. $^{7}[\alpha]_{\mathrm{D}}^{23}-51.9\left(c 1.04, \mathrm{CHCl}_{3}\right), 97 \%$ e.e. $\left.(S)\right)$, $90 \%$ e.e. $(R)$ by GC (Chiracel $\beta$-DEX 120, T $=125^{\circ} \mathrm{C}, \mathrm{P}=11 \mathrm{psi}, R$ isomer $24.2 \mathrm{~min}, S$ isomer $25.2 \mathrm{~min}$ ).

\section{H) Synthesis of $(1 R, 2 S)$-(N-3-cyclohexa-1,4-dienyl-propyl)-norephedrine 5}

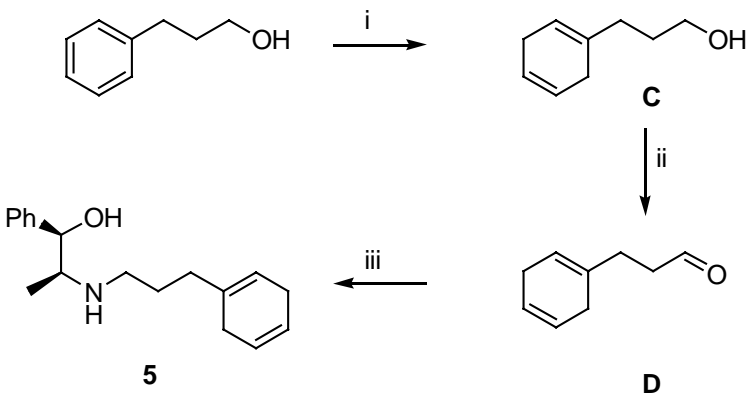

(i) $\mathrm{Na}, \mathrm{NH}_{3}, \mathrm{EtOH},-78^{\circ} \mathrm{C}, 3 \mathrm{~h}$; (ii) $(\mathrm{ClCO})_{2}(1.2 \mathrm{eq})$, DMSO (2.5 eq), $\mathrm{Et}_{3} \mathrm{~N}$ (5.5 eq), DCM, $-78^{\circ} \mathrm{C}, 80 \mathrm{~min}$; (iii) (a) 4 $\AA$ molecular sieves, L-(-)-norephedrine (1 eq), DCM, rt, o/n, (b) $\mathrm{NaBH}_{4}$ (3 eq), $\mathrm{MeOH}, \mathrm{rt}, \mathrm{o} / \mathrm{n}$; EtOH: ethanol; DMSO: dimethylsulfoxide; rt: room temperature; o/n: overnight.

\section{1) Synthesis of 3-cyclohexa-1,4-dienyl-propan-1-ol C}

To a refluxing solution of ammonia ${ }^{2}(350 \mathrm{~mL})$ was slowly added a solution of 3-phenyl-propan-1-ol (10 g, $\left.73 \mathrm{mmol}\right)$ in ethanol $(24 \mathrm{~mL})$ at $-78^{\circ} \mathrm{C}$ while stirring. Small pieces of cleaned sodium were added to the reaction mixture until the blue coloured persisted. During the addition of sodium, a small amount of ethanol was several times added to facilitate stirring. After the addition of sodium over the course of $3 \mathrm{~h}$, the reaction mixture was carefully poured into an ice-cooled solution of ammonium chloride. 3-cyclohexa-1,4-dienyl-propan-1-ol C was then extracted with dichloromethane $(3 \times 25 \mathrm{~mL})$ and the combined organic layer was dried over magnesium sulfate, filtered and concentrated under reduced pressure to afford a colourless oil. The oil was purified by bulb-to-bulb distillation under reduced pressure $\left(5.10^{-2}-3.10^{-2} \mathrm{~mm} \mathrm{Hg}, 100-105{ }^{\circ} \mathrm{C}\right)(10 \mathrm{~g}, 100 \%$ yield $)$. IR $v_{\max }\left(\mathrm{cm}^{-1}\right) 3309,3024,2936,2850$, 2818, 1428 and 1057; ${ }^{1} \mathrm{H}$ NMR $\left(400 \mathrm{MHz} \mathrm{CDCl}_{3}\right) \delta_{\mathrm{H}}(\mathrm{ppm}) 5.71$ (br s, 2H), 5.45 (br s, 1H), 3.65-3.62 (m, 2H) and 2.68-2.66 (m, 2H), 2.62-2.58 (m, 2H), $2.04(\mathrm{t}, J=7.6 \mathrm{~Hz}, 2 \mathrm{H}), 1.88(\mathrm{br} \mathrm{s}, 1 \mathrm{H})$ and $1.73-1.66(\mathrm{~m}, 2 \mathrm{H}) ;{ }^{13} \mathrm{C} \mathrm{NMR}(100$ 
$\left.\mathrm{MHz}, \mathrm{CDCl}_{3}\right) \delta_{\mathrm{C}}(\mathrm{ppm}) 134.9,124.65,124.6,119.1,63.1,34.1,30.6,29.3$ and 27.1; MS (EI) m/z 138 ([M] $\left.]^{+}, 17\right)$, 120 (18), 105 (16), 91 (100), 79 (61) and 67 (7); HRMS (EI) m/z calculated for $\mathrm{C}_{9} \mathrm{H}_{14} \mathrm{O} 138.1044$. Found 138.1042.

\section{2) Synthesis of 3-cyclohexa-1,4-dienyl-propionaldehyde D}

To a solution of oxalylchloride at $-78^{\circ} \mathrm{C}(0.75 \mathrm{~mL}, 8.7 \mathrm{mmol})$ in dichloromethane $(9 \mathrm{~mL})$ was added dropwise a solution of dimethylsulfoxide $(1.3 \mathrm{~mL}, 18 \mathrm{mmol})$ in dichloromethane $(9 \mathrm{~mL})$. The solution was stirred $15 \mathrm{minutes}$ at $-78^{\circ} \mathrm{C}$ then a solution of 3-cyclohexa-1,4-dienyl-propan-1-ol C (1 g, $\left.7 \mathrm{mmol}\right)$ in dichloromethane (18 $\left.\mathrm{mL}\right)$ was slowly added at the same temperature. After 50 minutes of stirring at $-78^{\circ} \mathrm{C}$, triethylamine $(5.5 \mathrm{~mL}, 40 \mathrm{mmol}) \mathrm{was}$ added and the reaction mixture was allowed to warm up to room temperature. After 15 minutes, water $(20 \mathrm{~mL})$ was added and the aldehyde was extracted with dichloromethane $(3 \times 15 \mathrm{~mL})$. The combined organic layer was dried over sodium sulfate, filtered and concentrated under reduced pressure to give slightly clear green oil. The oil was purified by bulb-to-bulb distillation under reduced pressure $\left(5.10^{-2}-3.10^{-2} \mathrm{~mm} \mathrm{Hg}, 70-80{ }^{\circ} \mathrm{C}\right)$ to afford a colourless oil $(0.9 \mathrm{~g}, 97 \%$ yield $)$. IR $v_{\max }\left(\mathrm{cm}^{-1}\right) 3026,2881,2850,2818,2718,1720$ and $1428 ;{ }^{1} \mathrm{H} \mathrm{NMR}\left(300 \mathrm{MHz}^{\mathrm{N}} \mathrm{CDCl}_{3}\right) \delta_{\mathrm{H}}$ (ppm) $9.78(\mathrm{~s}, 1 \mathrm{H}), 5.70$ (br s, 2H), 5.44 (br s, 1H), 2.72-2.62 (m, 2H), 2.62-2.54 (m, 4H) and $2.31(\mathrm{t}, J=7.4 \mathrm{~Hz}$, $2 \mathrm{H}) ;{ }^{13} \mathrm{C}$ NMR (75.5 MHz, CDCl 3$) \delta_{\mathrm{C}}(\mathrm{ppm}) 202.2,132.8,124.0,123.6,119.0,41.3,29.25,28.85$ and 26.4 ; MS (EI) $\mathrm{m} / \mathrm{z} 136\left([\mathrm{M}]^{+}, 8\right), 134(22), 118(78), 91(100), 79(70)$ and 65 (24); HRMS (EI) m/z calculated for $\mathrm{C}_{9} \mathrm{H}_{12} \mathrm{O}$ 136.0888. Found 136.0888.

\section{3) Synthesis of $(1 R, 2 S)$-(N-3-cyclohexa-1,4-dienyl-propyl)-norephedrine 5}

To a suspension of powdered $4 \AA$ molecular sieves $(2.8 \mathrm{~g})$ in dichloromethane (40 mL) was added 3-cyclohexa-1,4dienyl-propionaldehyde $\mathbf{D}(2.0 \mathrm{~g}, 15 \mathrm{mmol})$, followed by L-(-)-norephedrine $(2.2 \mathrm{~g}, 15 \mathrm{mmol})$ at room temperature. The reaction mixture was stirred overnight at room temperature, filtered and concentrated under reduced pressure. The colourless oily residue was dissolved in methanol and sodium borohydride (1.7 g, $45 \mathrm{mmol})$ was added at room temperature. The reaction mixture was stirred overnight and water $(20 \mathrm{~mL})$ was added. The product was extracted with dichloromethane ( $3 \times 20 \mathrm{~mL})$ and the combined organic layer dried over magnesium sulfate and filtered. After concentration under reduced pressure, a colourless oil was obtained and was purified by flash chromatography $\left(20 \rightarrow 80 \% \mathrm{v} / \mathrm{v}\right.$ ethyl acetate: hexane) to afford a white solid $(2.6 \mathrm{~g}, 65 \%$ yield $)$. mp $59-60{ }^{\circ} \mathrm{C} ;[\alpha]_{\mathrm{D}}{ }^{20}-5.6(c 0.9$, $\left.\mathrm{CHCl}_{3}\right)$; IR $v_{\max }\left(\mathrm{cm}^{-1}\right) 3082,3023,2970,2879,2853,2818$ and $1426 ;{ }^{1} \mathrm{H}$ NMR $\left(400 \mathrm{MHz}, \mathrm{CDCl}_{3}\right) \delta_{\mathrm{H}}(\mathrm{ppm}) 7.33-$ $7.23(\mathrm{~m}, 5 \mathrm{H}), 5.71$ (br s, 2H), 5.44 (br s, 1H), 4.77-7.76 (m, 1H), 2.98-2.92 (m, 1H), 2.79-2.58 (m, 6H), 2.34 (br s, $1 \mathrm{H}), 2.05-2.01(\mathrm{~m}, 2 \mathrm{H}), 1.68-1.61(\mathrm{~m}, 2 \mathrm{H})$ and $0.83(\mathrm{~d}, J=6.5 \mathrm{~Hz}, 3 \mathrm{H}) ;{ }^{13} \mathrm{C} \mathrm{NMR}\left(100 \mathrm{MHz} \mathrm{CDCl}_{3}\right) \delta_{\mathrm{C}}(\mathrm{ppm})$ $141.7,134.8,128.5,127.5,126.5,124.7,124.6,119.2,73.4,58.9,47.2,35.5,29.3,28.0,27.2$ and 14.8; MS (EI) m/z $272\left([\mathrm{M}+\mathrm{H}]^{+}, 3\right), 252(48), 164(100), 91(55), 79$ (22) and 58 (17); Anal. Calculated for $\mathrm{C}_{18} \mathrm{H}_{25} \mathrm{NO}: \mathrm{C}, 79.66 ; \mathrm{H}$, 9.28; N; 5.16. Found: C, 79.82; H, 9.40; N, 5.26.

\section{I) Synthesis of the chloro-bridged $\eta^{6}$-arene ruthenium (II) dimer $3^{4}$}

To a stirred solution of $(1 R, 2 S)$-(N-3-cyclohexa-1,4-dienyl-propyl)-norephedrine $\mathbf{5}(1.709 \mathrm{~g}, 6.3 \mathrm{mmol})$ in diethyl ether (20 mL) was added a $2 \mathrm{M}$ solution of hydrochloric acid in diethyl ether $(9 \mathrm{~mL}, 19 \mathrm{mmol})$ at room temperature. The reaction mixture was stirred 5 minutes at room temperature and concentrated under reduced pressure to give a white residue. To a suspension of the residue in ethanol $(50 \mathrm{~mL})$ was added hydrate ruthenium (III) trichloride (1.089 g, $5.2 \mathrm{mmol})$. The reaction mixture was stirred at reflux for $14 \mathrm{~h}$ and filtered to give green solid residue. The residue was washed with anhydrous dichloromethane to afford a green/khaki solid (1.358 g, 55\% yield). mp 239-240 ${ }^{\circ} \mathrm{C}$ (dec.); IR $v_{\max }\left(\mathrm{cm}^{-1}\right) 3291,3042,2980,2788,1448$ and $1410 ;{ }^{1} \mathrm{H} \mathrm{NMR}$ $(400 \mathrm{MHz}, \mathrm{DMSO}) \delta_{\mathrm{H}}(\mathrm{ppm}) 8.71(\mathrm{br} \mathrm{s}, 2 \mathrm{H}), 7.40-7.25(\mathrm{~m}, 5 \mathrm{H}), 6.11(\mathrm{~d}, J=4.0 \mathrm{~Hz}, 1 \mathrm{H}), 6.04(\mathrm{t}, J=5,9 \mathrm{~Hz}, 2 \mathrm{H}), 5.84(\mathrm{~d}, J=$ $5.5 \mathrm{~Hz}, 3 \mathrm{H}), 5.16$ (br s, $1 \mathrm{H}), 3.45$ (br s, 1H), 3.14 (br s, 2H), 2.64-2.60 (m, 2H), 2.09-2.02 (m, 2H) and $0.94(\mathrm{~d}, J=6.4 \mathrm{~Hz}, 3 \mathrm{H})$; ${ }^{13} \mathrm{C}$ NMR (100 MHz, DMSO) $\delta_{\mathrm{C}}(\mathrm{ppm}) 141.2,128.15,127.25,125.8,106.1,88.8,85.4,83.7,69.3,58.3,44.1,29.5,25.0$ and 9.3; HRMS (LSIMS) $\mathrm{m} / \mathrm{z}$ calculated for ${ }^{102} \mathrm{RuC}_{18} \mathrm{H}_{24} \mathrm{NOCl}_{2}$ 442.0278. Found 442.0290. Dimer was not observed by LSIMS or ESIMS. 
J) General experimental procedure for the asymmetric transfer hydrogenation catalysed by ruthenium (II) dimer 3

To a stirred suspension of ruthenium (II) dimer $3\left(0.004 \mathrm{~g}, 4.2 .10^{-3} \mathrm{mmol}\right)$ in dry and degassed propan-2-ol (15 $\left.\mathrm{mL}\right)$ was added a $0.1 \mathrm{M}$ solution of $\mathrm{KOH}\left(0.84 \mathrm{~mL}, 8.5 .10^{-2} \mathrm{mmol}\right)$, followed by ketone $(1.7 \mathrm{mmol})$ at $28^{\circ} \mathrm{C}$. The reaction mixture was stirred at $28^{\circ} \mathrm{C}$ until completion and hexane was added $(30 \mathrm{~mL})$. The mixture was filtered through a pad of silica gel and the pad was washed with a $40 \%$ solution of ethyl acetate in hexane $(150 \mathrm{~mL})$. The filtrate was concentrated under reduced pressure to afford the reduction product. Purification by flash chromatography was applied when appropriate.

\section{K) HPLC, GC analysis and optical rotation data of the reduction products}

(R)-1-phenylethanol: $[\alpha]_{\mathrm{D}}{ }^{28}+25.8\left(c 1.00, \mathrm{CHCl}_{3}\right)\left(\right.$ lit. $^{9}[\alpha]_{\mathrm{D}}{ }^{23}+48.6\left(c 1.0, \mathrm{CH}_{2} \mathrm{Cl}_{2}\right), 96 \%$ e.e. $\left.(R)\right)$, $66 \%$ e.e. $(R)$ by GC (Chiracel $\beta$-DEX 120, T $=115^{\circ} \mathrm{C}, \mathrm{P}=11 \mathrm{psi}, R$ isomer $9.0 \mathrm{~min}, S$ isomer $9.5 \mathrm{~min}$ ).

(R)-1-(4'-methylphenyl)ethanol: $[\alpha]_{\mathrm{D}}{ }^{28}+31.8\left(c 1.00, \mathrm{CHCl}_{3}\right)\left(\right.$ lit. ${ }^{0}[\alpha]_{\mathrm{D}}-54.1\left(c 0.0532, \mathrm{CHCl}_{3}\right), 96 \%$ e.e. $\left.(S)\right)$, $57 \%$ e.e. $(R)$ by GC (Chiracel $\beta$-DEX $120, \mathrm{~T}=115^{\circ} \mathrm{C}, \mathrm{P}=11$ psi, $R$ isomer $13.2 \mathrm{~min}, S$ isomer $14.1 \mathrm{~min}$ ).

(R)-1-(4'-chlorophenyl)ethanol: $[\alpha]_{\mathrm{D}}{ }^{28}+24.8\left(c 1.00, \mathrm{CHCl}_{3}\right)\left(\right.$ lit. $^{9}[\alpha]_{\mathrm{D}}{ }^{21}+46.1(c 0.9-1.1$, ether $), 91 \%$ e.e. $\left.(R)\right), 54$ $\%$ e.e. $(R)$ by $\mathrm{GC}$ (Chiracel $\beta$-DEX $120, \mathrm{~T}=140^{\circ} \mathrm{C}, \mathrm{P}=11 \mathrm{psi}, R$ isomer $11.1 \mathrm{~min}, S$ isomer $\left.11.7 \mathrm{~min}\right)$.

(R)-1-(4'-methoxyphenyl)ethanol: $[\alpha]_{\mathrm{D}}{ }^{28}+28.0\left(c 1.00, \mathrm{CHCl}_{3}\right)\left(\right.$ lit. $^{7}[\alpha]_{\mathrm{D}}{ }^{23}-51.9\left(c 1.04, \mathrm{CHCl}_{3}\right), 97 \%$ e.e. $\left.(S)\right)$, $52 \%$ e.e. $(R)$ by GC (Chiracel $\beta$-DEX $120, \mathrm{~T}=125^{\circ} \mathrm{C}, \mathrm{P}=11 \mathrm{psi}, R$ isomer $25.1 \mathrm{~min}, S$ isomer $25.9 \mathrm{~min}$ ).

(R)-1-(4'-fluorophenyl)ethanol: $[\alpha]_{\mathrm{D}}{ }^{28}+26.4\left(c 1.00, \mathrm{CHCl}_{3}\right)\left(\right.$ lit. $^{10}[\alpha]_{\mathrm{D}}-47.5\left(c 0.0576, \mathrm{CHCl}_{3}\right), 97 \%$ e.e. $\left.(S)\right), 55$ $\%$ e.e. $(R)$ by GC (Chiracel $\beta$-DEX $120, \mathrm{~T}=115^{\circ} \mathrm{C}, \mathrm{P}=11 \mathrm{psi}, R$ isomer $10.1 \mathrm{~min}, S$ isomer $10.8 \mathrm{~min}$ ).

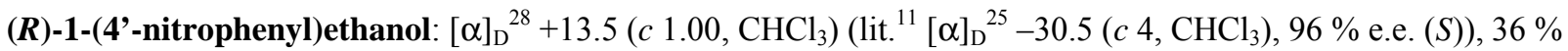
e.e. $(R)$ by $\mathrm{GC}$ (Chiracel $\beta$-DEX $120, \mathrm{~T}=170^{\circ} \mathrm{C}, \mathrm{P}=11 \mathrm{psi}, R$ isomer $18.7 \mathrm{~min}, S$ isomer $\left.19.4 \mathrm{~min}\right)$.

(R)-1-indanol: $[\alpha]_{\mathrm{D}}{ }^{28}-21.8\left(c 1.00, \mathrm{CHCl}_{3}\right)\left(\right.$ lit. $^{7}[\alpha]_{\mathrm{D}}{ }^{23}+30.9\left(c 1.99, \mathrm{CHCl}_{3}\right), 92 \%$ e.e. $\left.(S)\right), 66 \%$ e.e. $(R)$ by HPLC (chiralcel OD column, propan-2-ol:hexane = 2:98 $(1 \mathrm{~mL} / \mathrm{min}), S$ isomer $12.1 \mathrm{~min}, R$ isomer $13.7 \mathrm{~min})$.

(R)-1-(2'-naphthyl)ethanol: $[\alpha]_{\mathrm{D}}{ }^{28}+28.8\left(c 1.00, \mathrm{CHCl}_{3}\right)\left(\right.$ lit. $\left.^{8}[\alpha]_{\mathrm{D}}-34.3(c 1.10, \mathrm{EtOH})\right), 86 \%$ e.e. $\left.(S)\right), 58 \%$ e.e. $(R)$ by HPLC (chiralcel OD column, propan-2-ol:hexane $=2: 98(1 \mathrm{~mL} / \mathrm{min}), S$ isomer $21.7 \mathrm{~min}, R$ isomer $23.6 \mathrm{~min})$. 
L) ${ }^{1} \mathrm{H}$ and ${ }^{13} \mathrm{C}$ NMR spectra

1) ${ }^{1} \mathrm{H}$ and ${ }^{13} \mathrm{C}$ NMR spectra of 2-phenyl-ethanesulfonic acid $A$

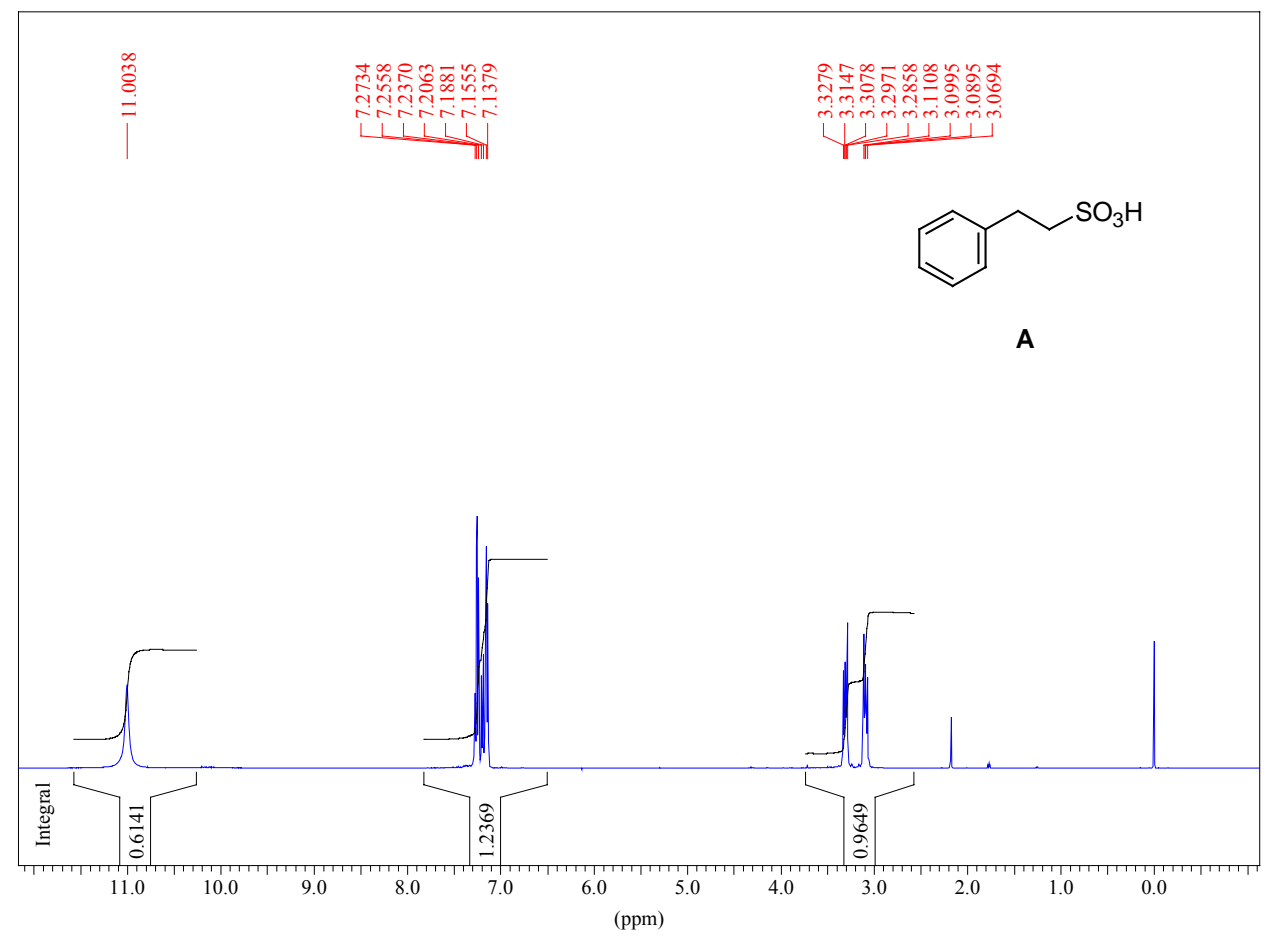

Parameters: Proton with $\mathrm{CDCl} 3 \quad \mathrm{SF}=400.03 \quad \mathrm{SW}=8223.68 \quad \mathrm{AQ}=3.98 \quad \mathrm{TD}=65536 \quad$ Units=ppm

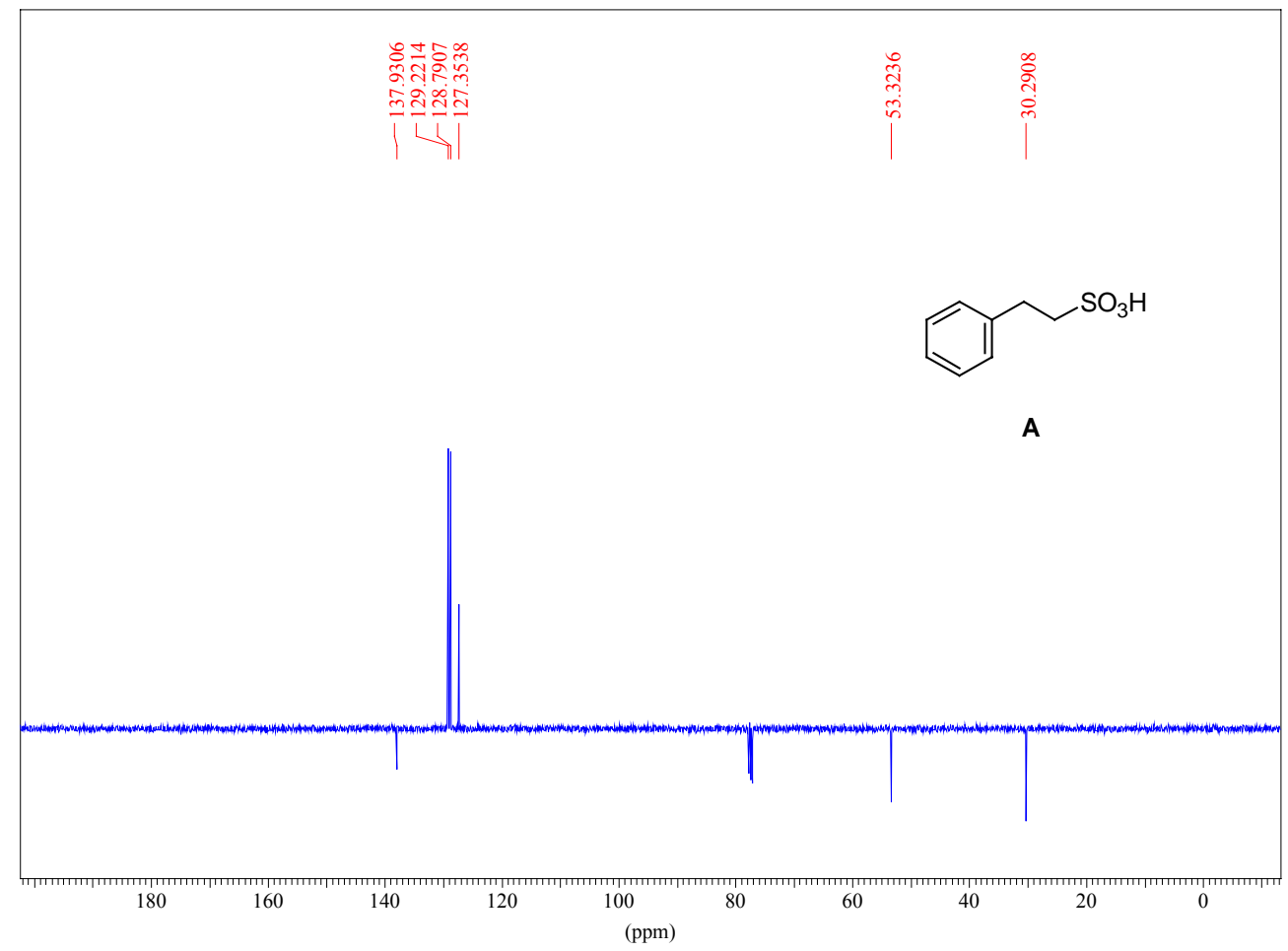

Parameters: C13pend. with $\mathrm{CDCl} 3 \quad \mathrm{SF}=100.59 \quad \mathrm{SW}=25125.63 \quad \mathrm{AQ}=1.30 \quad \mathrm{TD}=131072 \quad$ Units=ppm 
2) ${ }^{1} \mathrm{H}$ and ${ }^{13} \mathrm{C}$ NMR spectra of 2-cyclohexa-1,4-dienyl-ethanesulfonyl chloride B

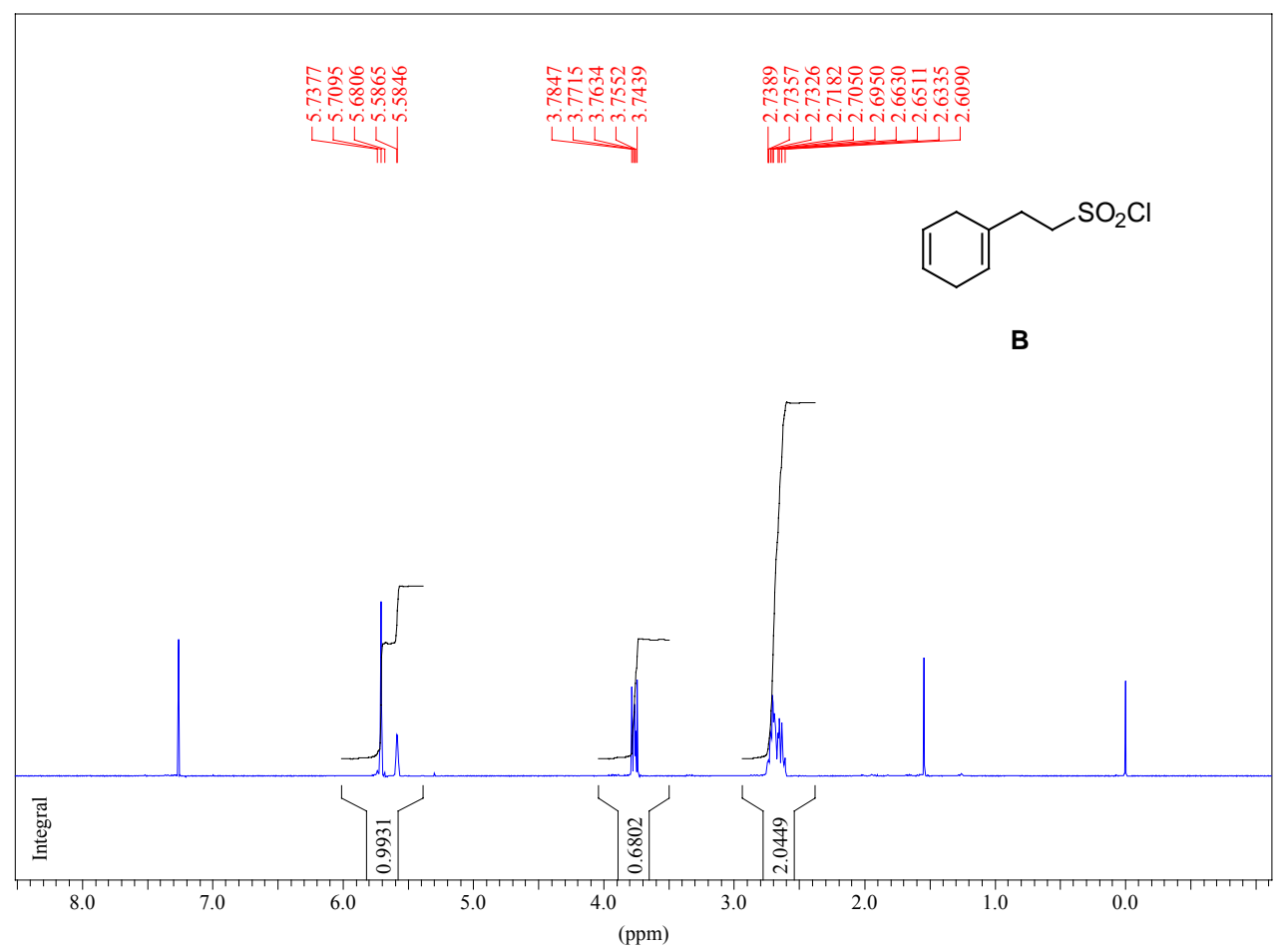

Parameters: Proton with $\mathrm{CDCl} 3 \quad \mathrm{SF}=400.03 \quad \mathrm{SW}=8223.68 \quad \mathrm{AQ}=1.99 \quad \mathrm{TD}=65536 \quad$ Units=ppm

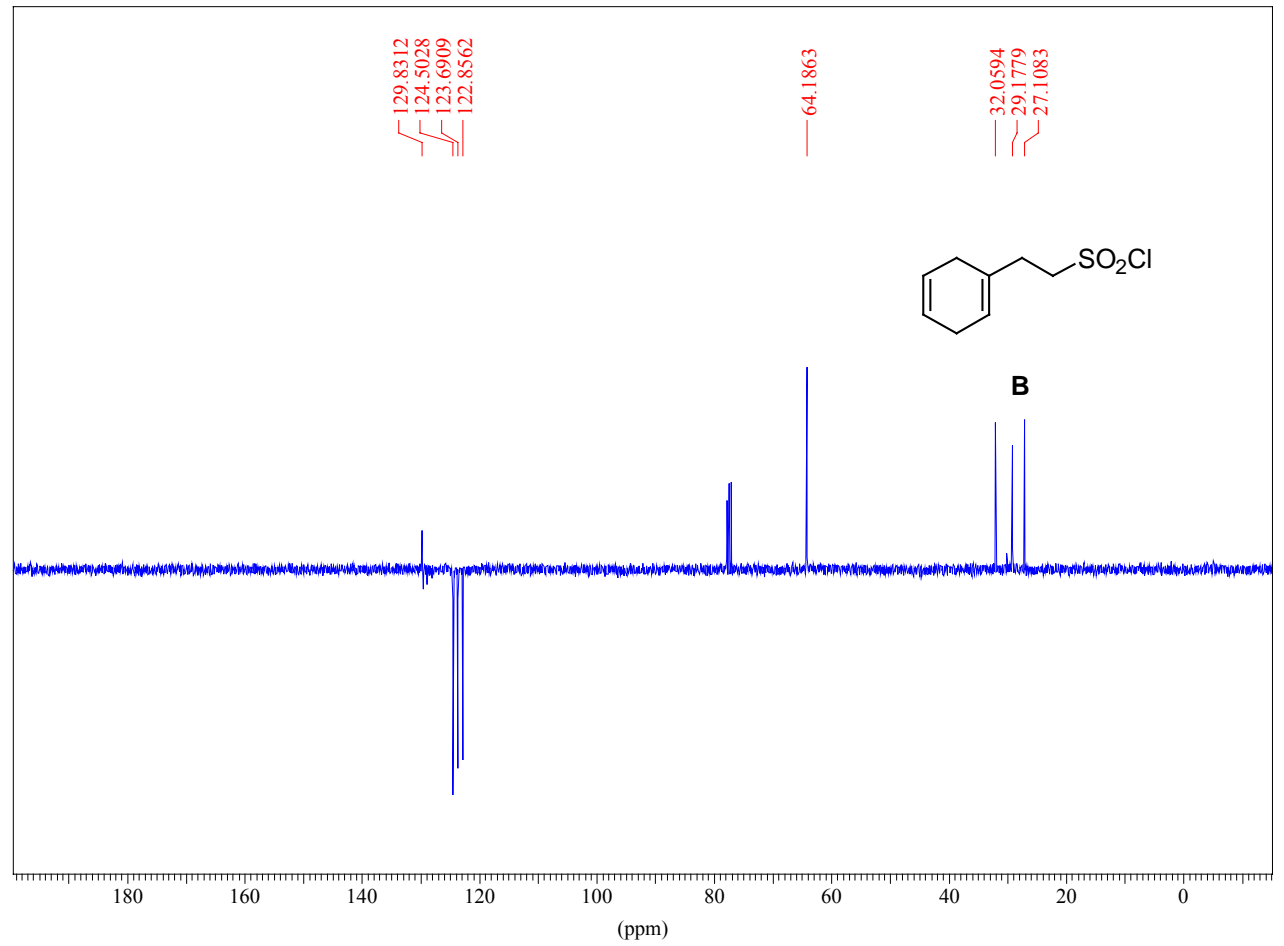

Parameters: C13pend. with $\mathrm{CDCl} 3 \quad \mathrm{SF}=100.59 \quad \mathrm{SW}=25125.63 \quad \mathrm{AQ}=1.30 \quad \mathrm{TD}=131072 \quad$ Units=ppm 
3) ${ }^{1} \mathrm{H}$ and ${ }^{13} \mathrm{C}$ NMR spectra of $(R, R)$-(N-2-cyclohexa-1,4-dienyl-ethanesulfonyl)-1,2-diphenylethylene-diamine 6

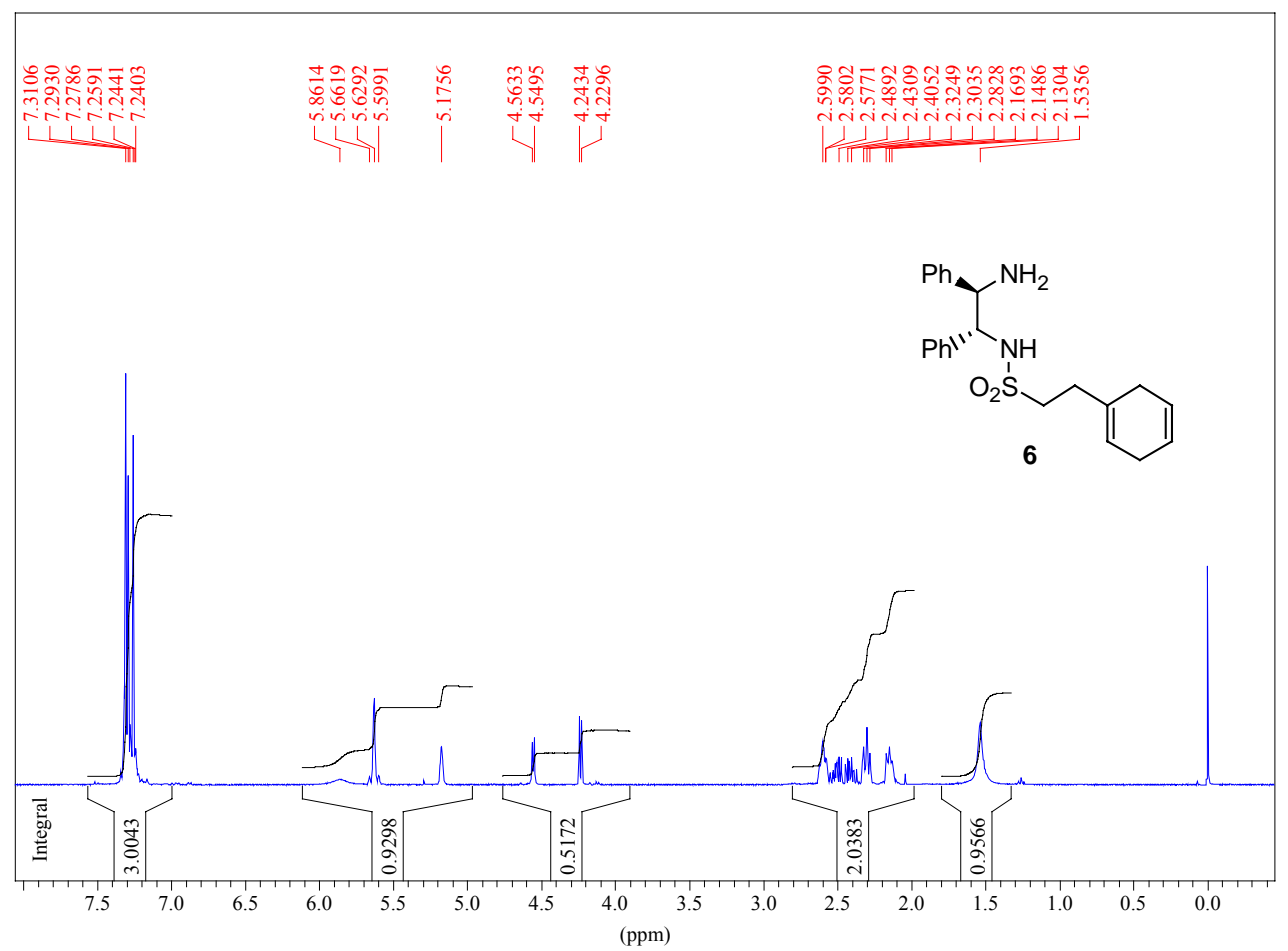

Parameters: Proton with $\mathrm{CDCl} 3 \quad \mathrm{SF}=400.03 \quad \mathrm{SW}=8223.68 \quad \mathrm{AQ}=1.99 \quad \mathrm{TD}=65536 \quad$ Units=ppm

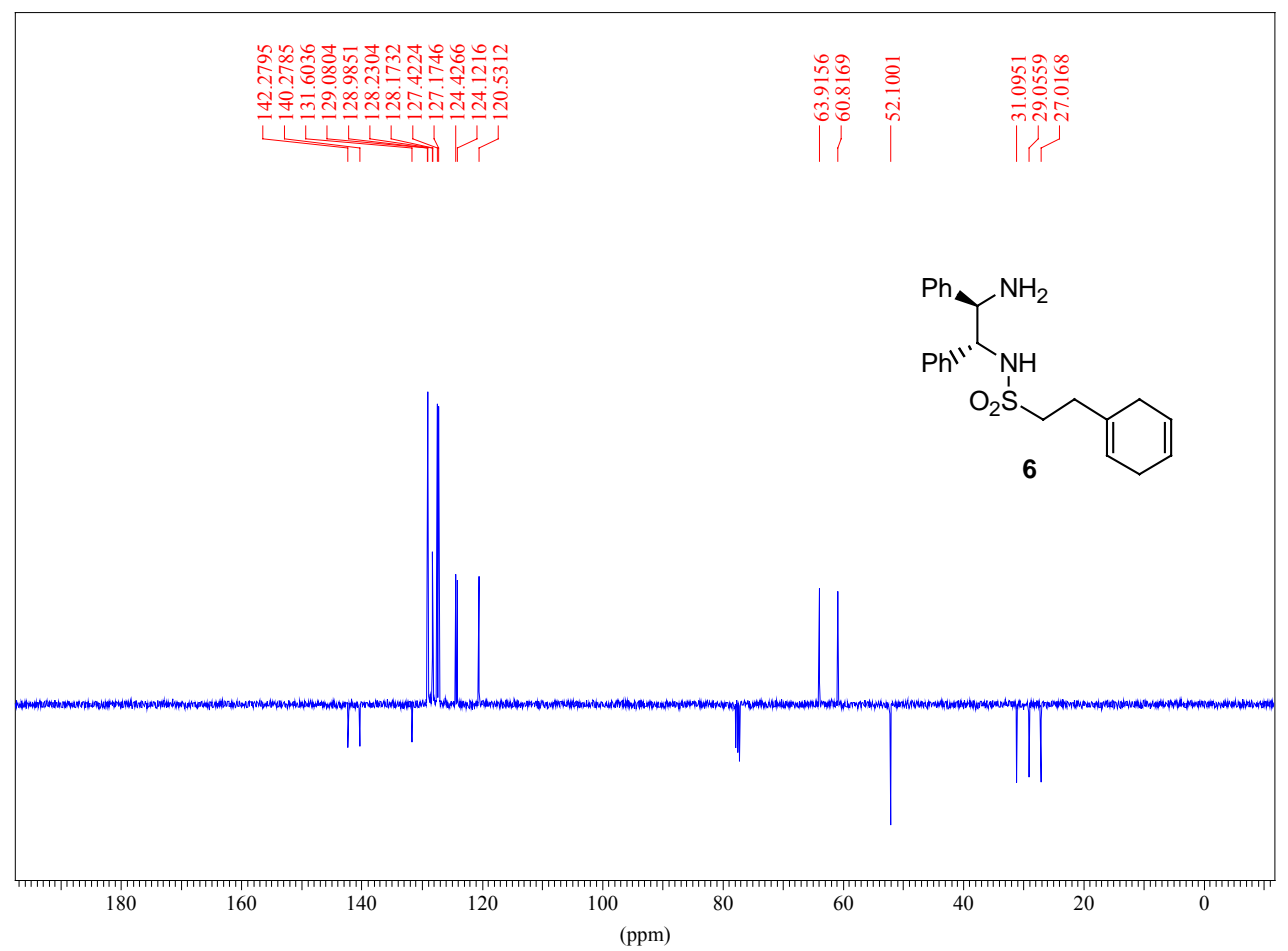

Parameters: C13pend.with CDCl3 $\quad \mathrm{SF}=100.59 \quad \mathrm{SW}=25125.63 \quad \mathrm{AQ}=1.30 \quad \mathrm{TD}=131072 \quad$ Units=ppm 

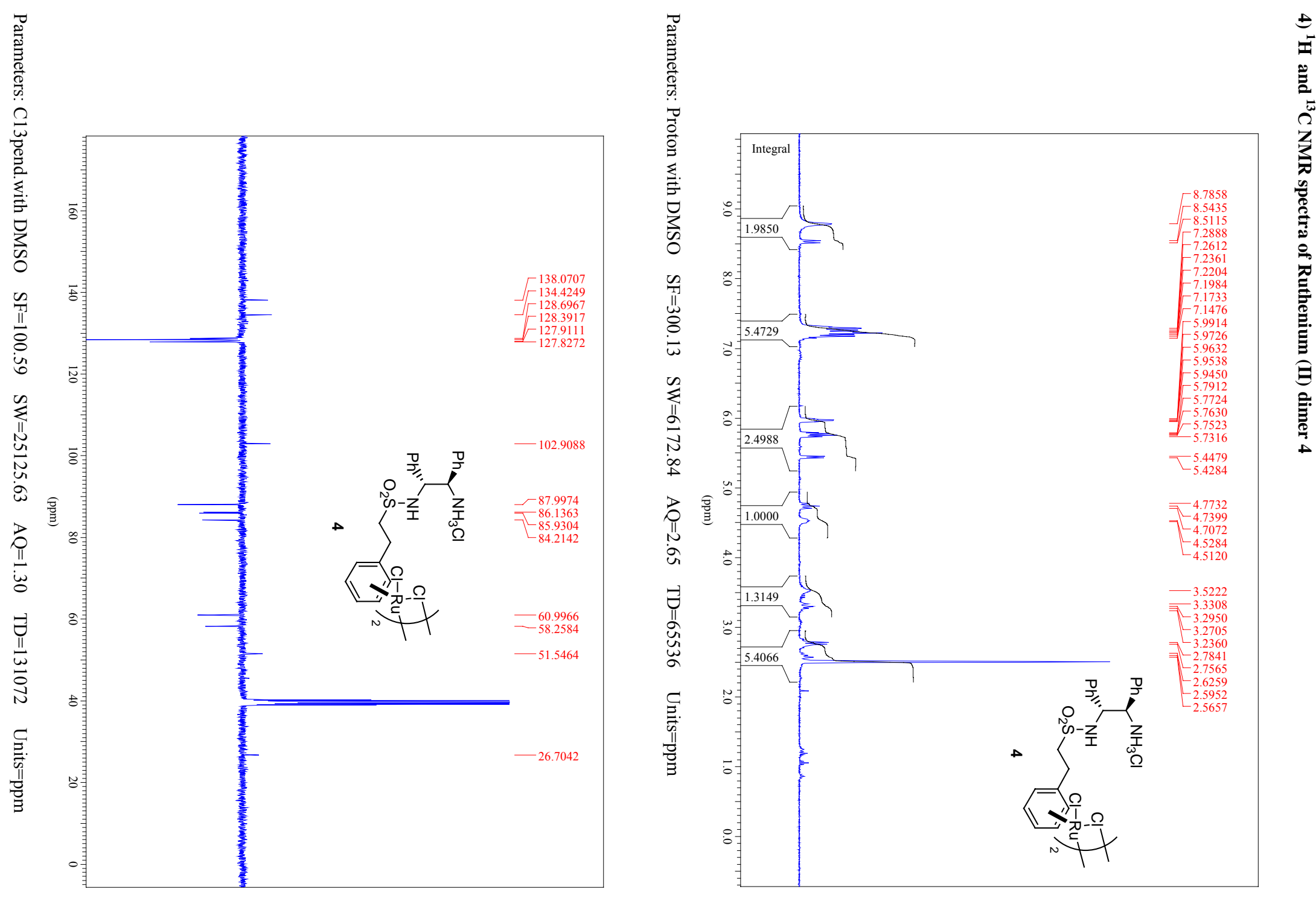


\section{5) ${ }^{1} \mathrm{H}$ and ${ }^{13} \mathrm{C}$ NMR spectra of 3-cyclohexa-1,4-dienyl-propan-1-ol C}

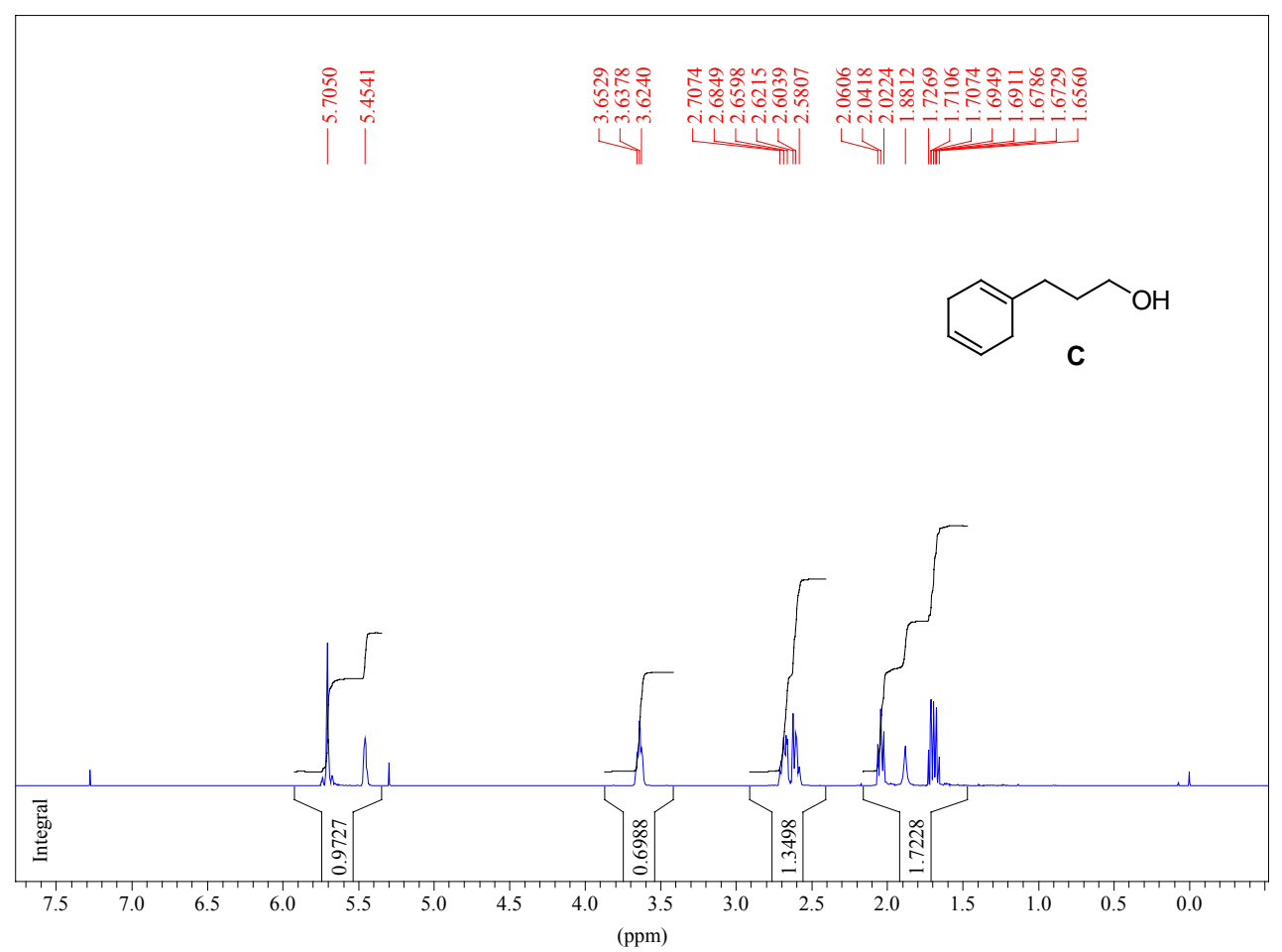

Parameters: Proton with $\mathrm{CDCl} 3 \quad \mathrm{SF}=400.03 \quad \mathrm{SW}=8223.68 \quad \mathrm{AQ}=1.99 \quad \mathrm{TD}=65536 \quad$ Units=ppm

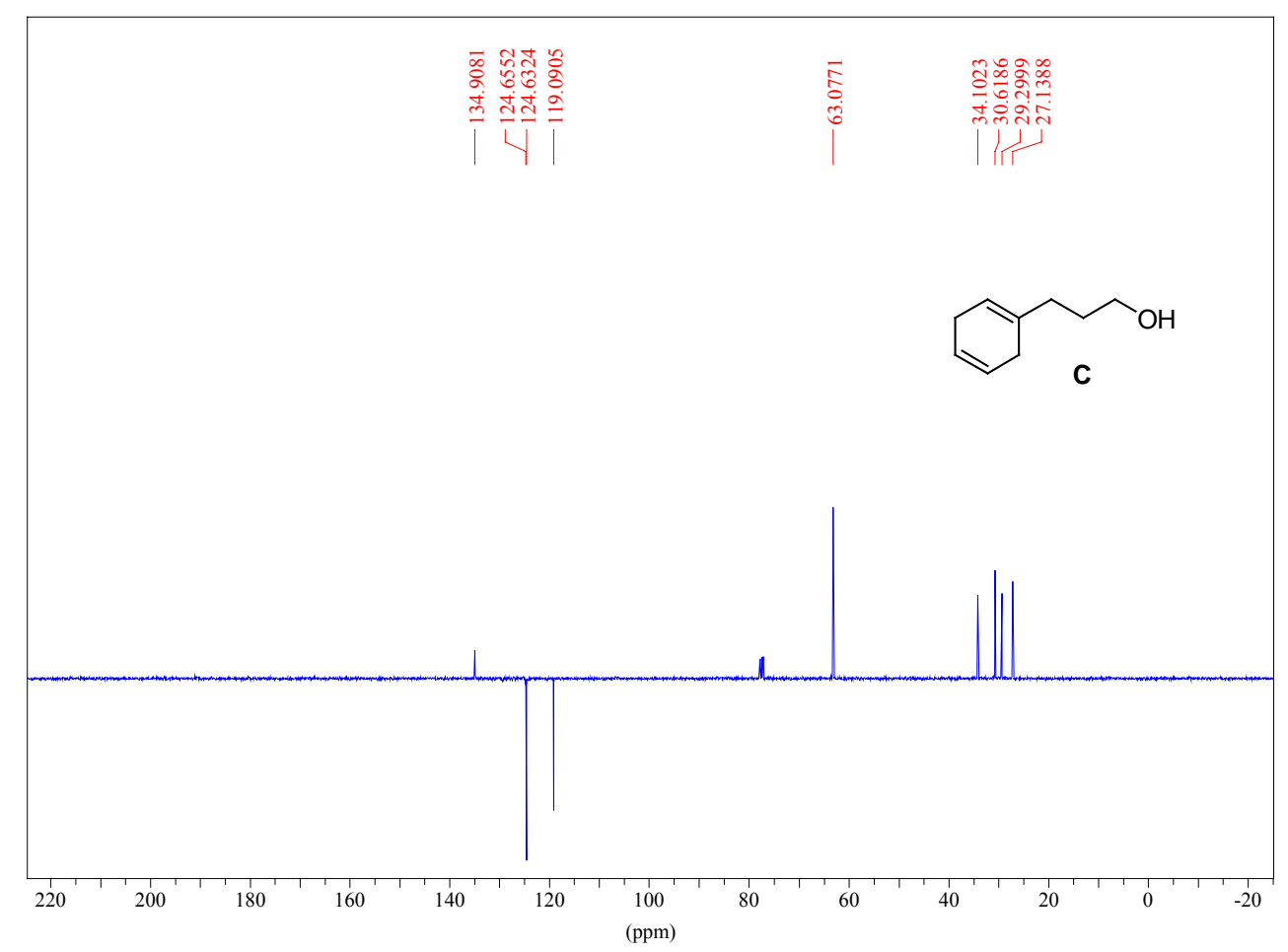

Parameters: C13pend. with $\mathrm{CDCl} 3 \quad \mathrm{SF}=100.59 \quad \mathrm{SW}=25125.63 \quad \mathrm{AQ}=1.30 \quad \mathrm{TD}=131072 \quad$ Units=ppm 
6) ${ }^{1} \mathrm{H}$ and ${ }^{13} \mathrm{C}$ NMR spectra of 3-cyclohexa-1,4-dienyl-propionaldehyde D

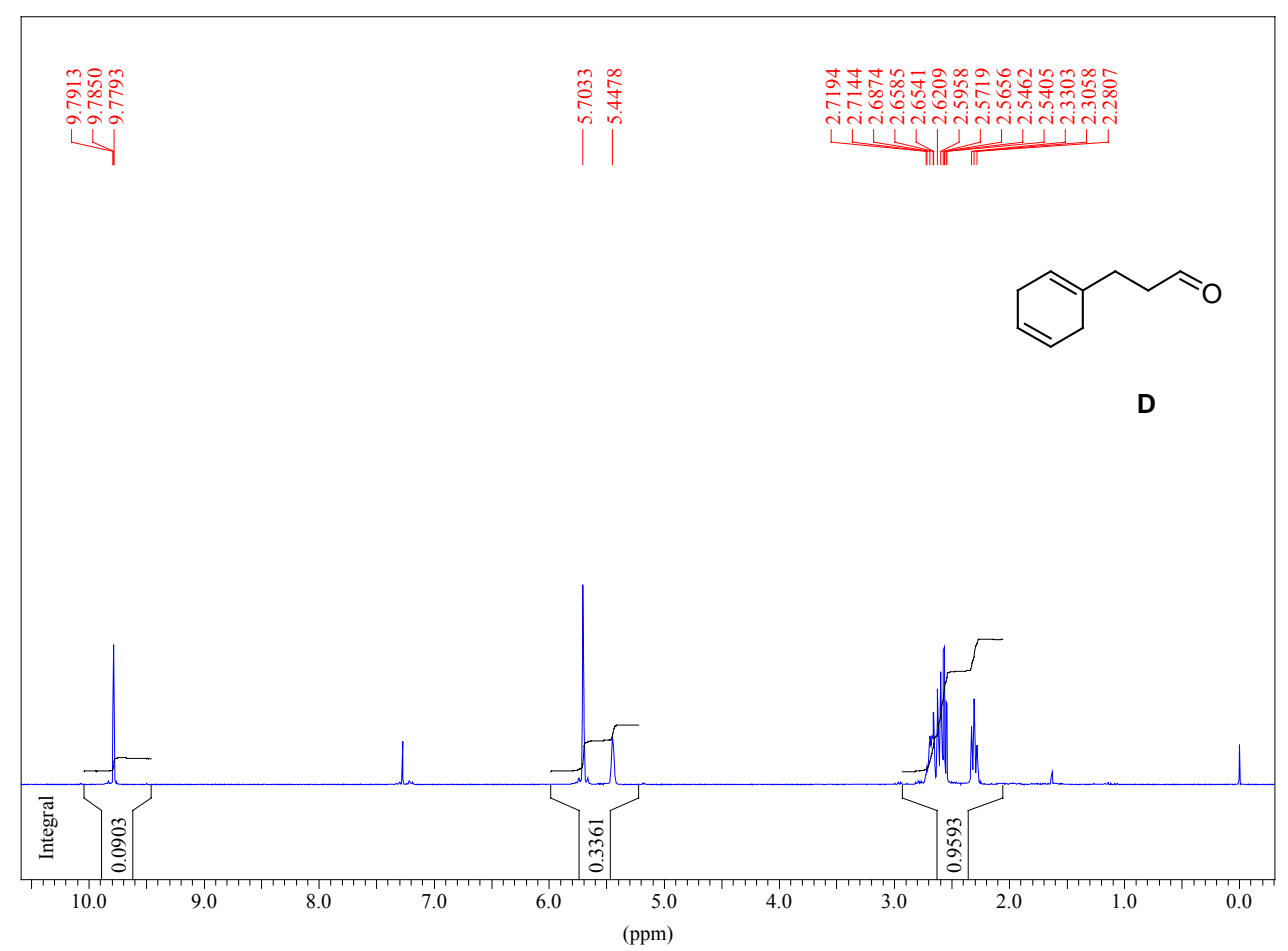

Parameters: Proton with $\mathrm{CDCl} 3 \quad \mathrm{SF}=300.13 \quad \mathrm{SW}=6172.84 \quad \mathrm{AQ}=2.65 \quad \mathrm{TD}=65536 \quad$ Units=ppm

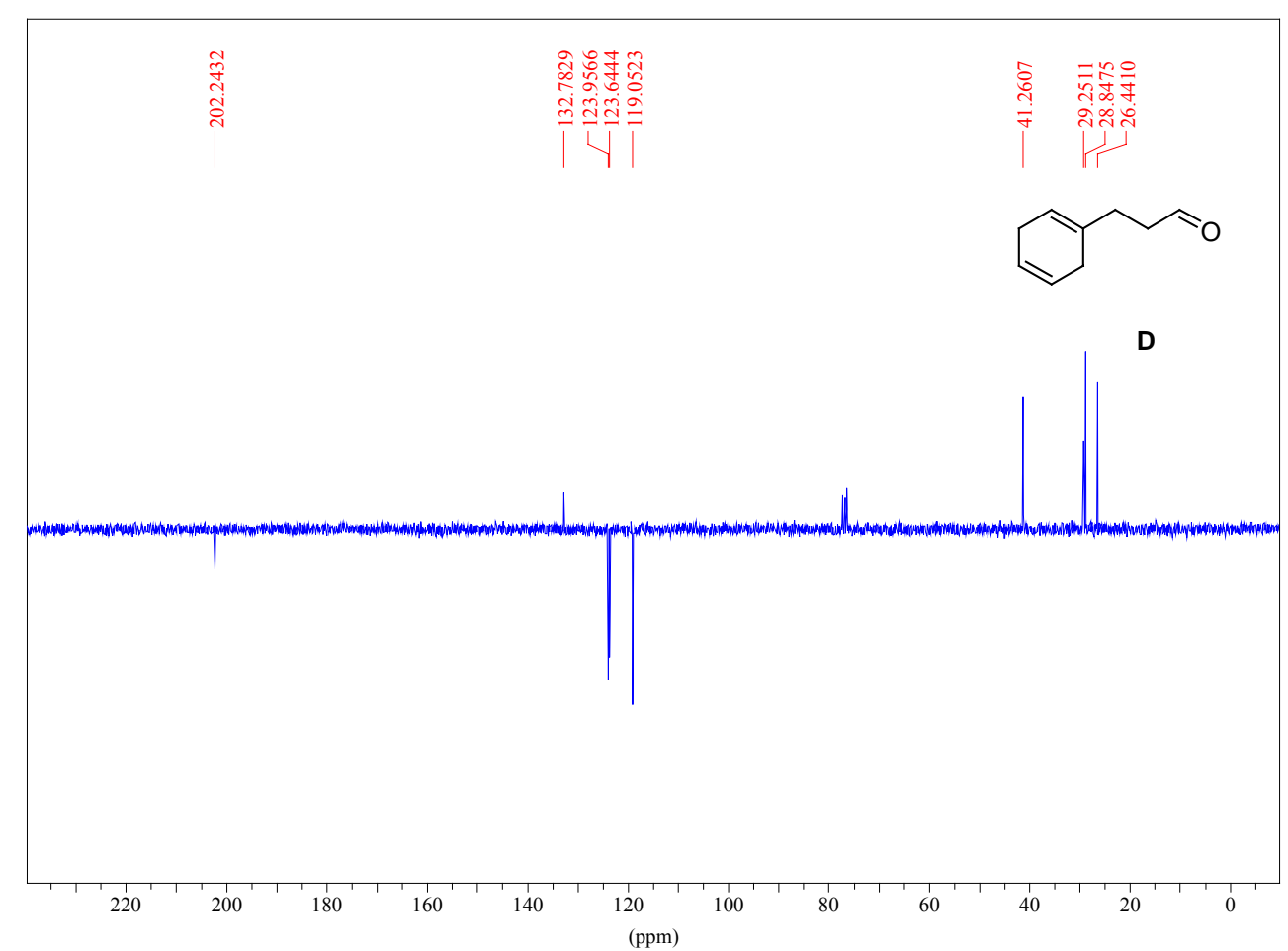

Parameters: C13pend. with $\mathrm{CDCl} 3 \quad \mathrm{SF}=75.47 \quad \mathrm{SW}=18832.39 \quad \mathrm{AQ}=1.74 \quad \mathrm{TD}=131072 \quad$ Units=ppm 


\section{7) ${ }^{1} \mathrm{H}$ and ${ }^{13} \mathrm{C}$ NMR spectra of $(1 R, 2 S)$-(N-3-cyclohexa-1,4-dienyl-propyl)-norephedrine 5}

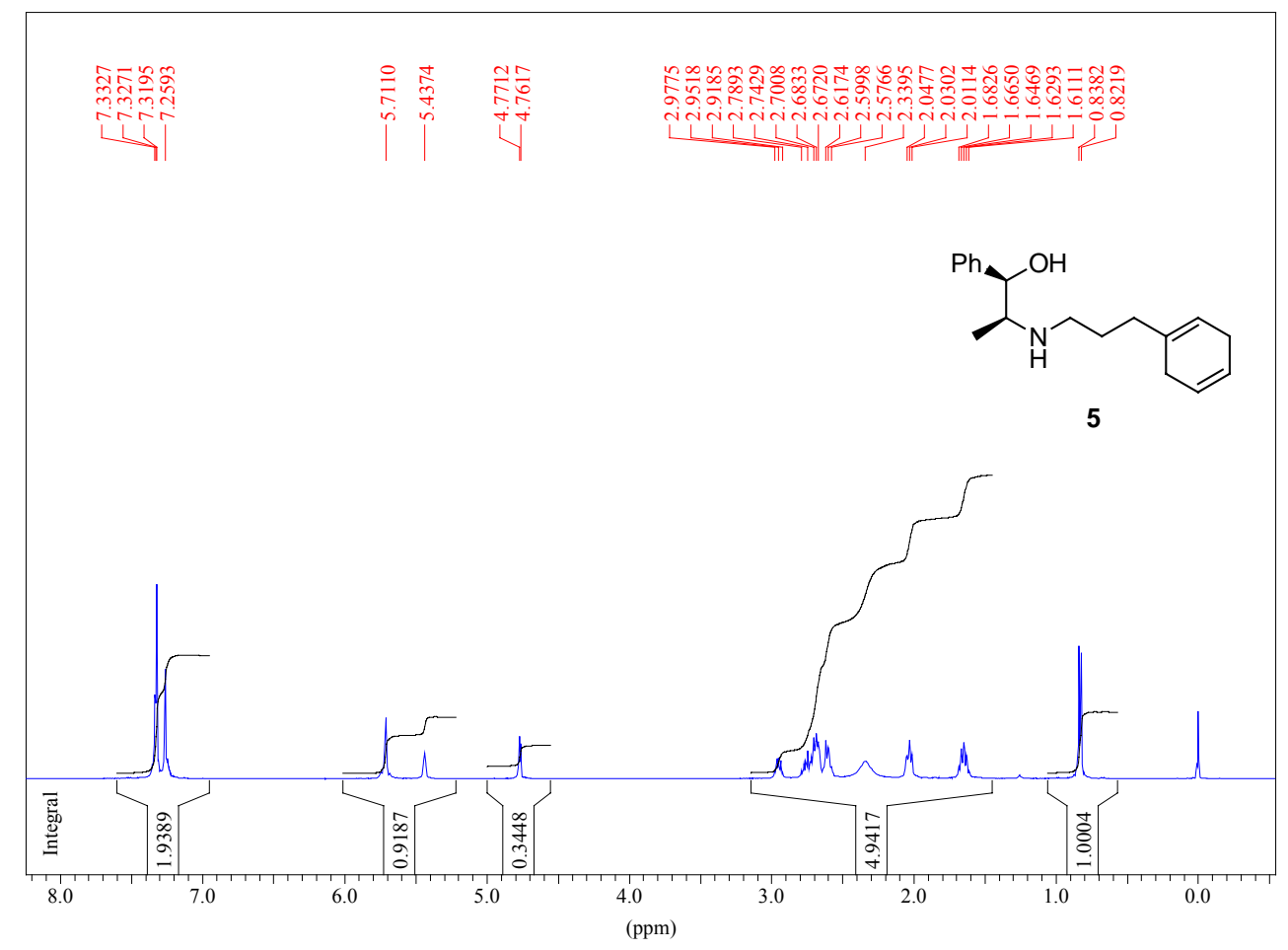

Parameters: Proton with $\mathrm{CDCl} 3 \quad \mathrm{SF}=400.03 \quad \mathrm{SW}=8223.68 \quad \mathrm{AQ}=1.99 \quad \mathrm{TD}=65536 \quad$ Units=ppm

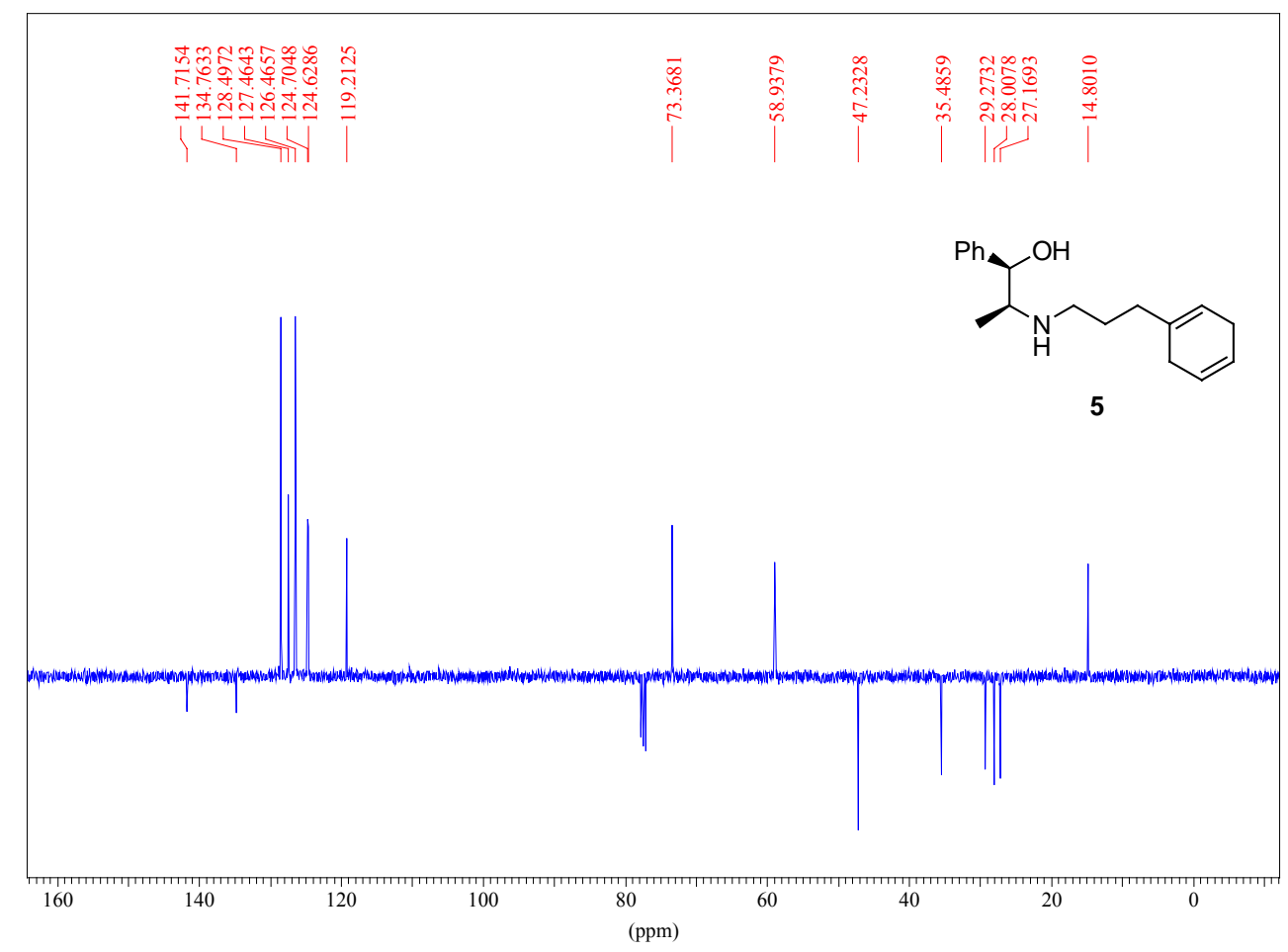

Parameters: $\mathrm{C} 13$ pend. with $\mathrm{CDCl} 3 \quad \mathrm{SF}=100.59 \quad \mathrm{SW}=25125.63 \quad \mathrm{AQ}=1.30 \quad \mathrm{TD}=131072 \quad$ Units=ppm 
8) ${ }^{1} \mathrm{H}$ and ${ }^{13} \mathrm{C}$ NMR spectra of chloro-bridged $\eta^{6}$-arene ruthenium (II) dimer 3

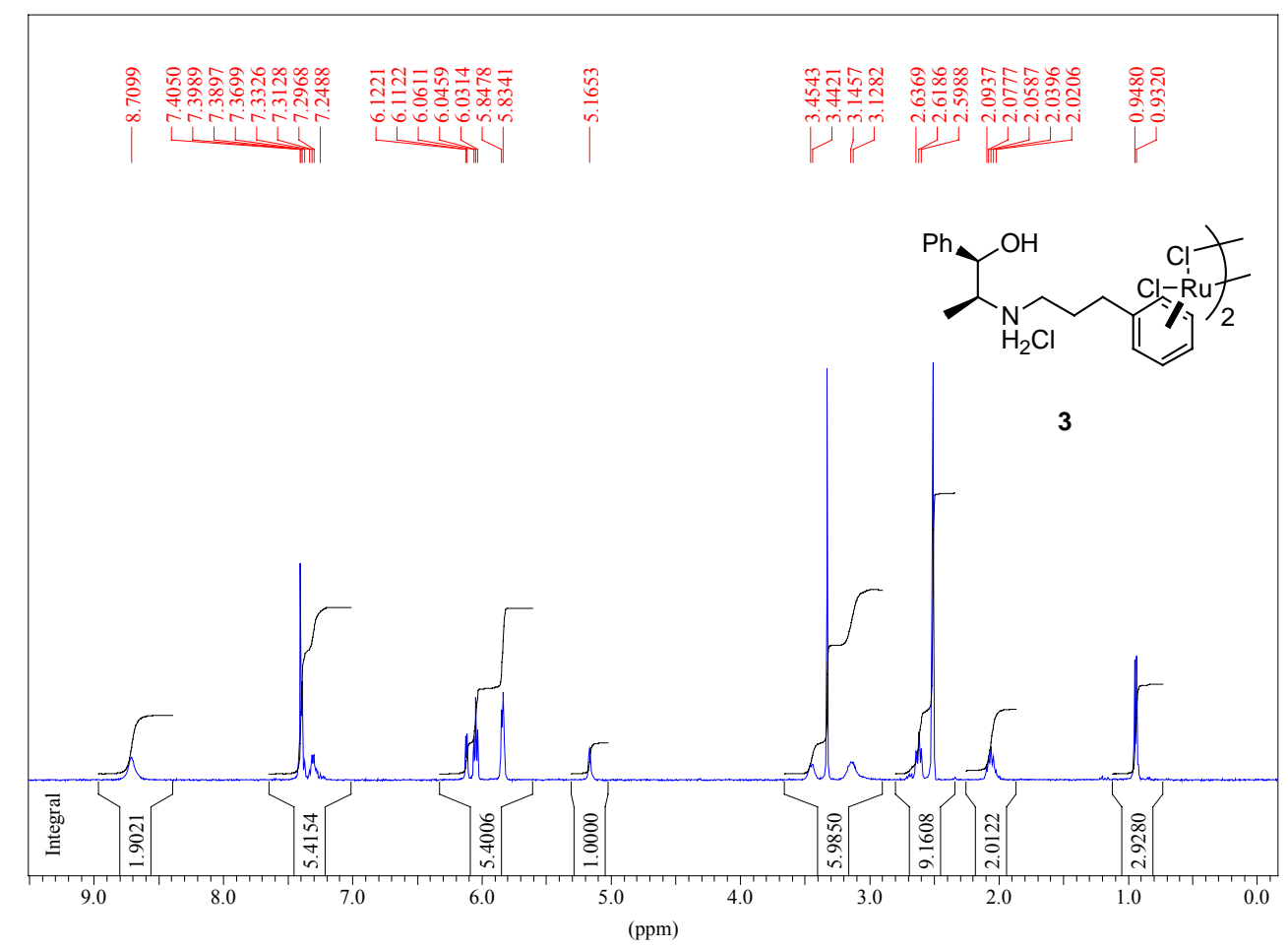

Parameters: Proton with DMSO $\quad \mathrm{SF}=399.80 \quad \mathrm{SW}=9980.04 \quad \mathrm{AQ}=1.64 \quad \mathrm{TD}=65536 \quad$ Units=ppm

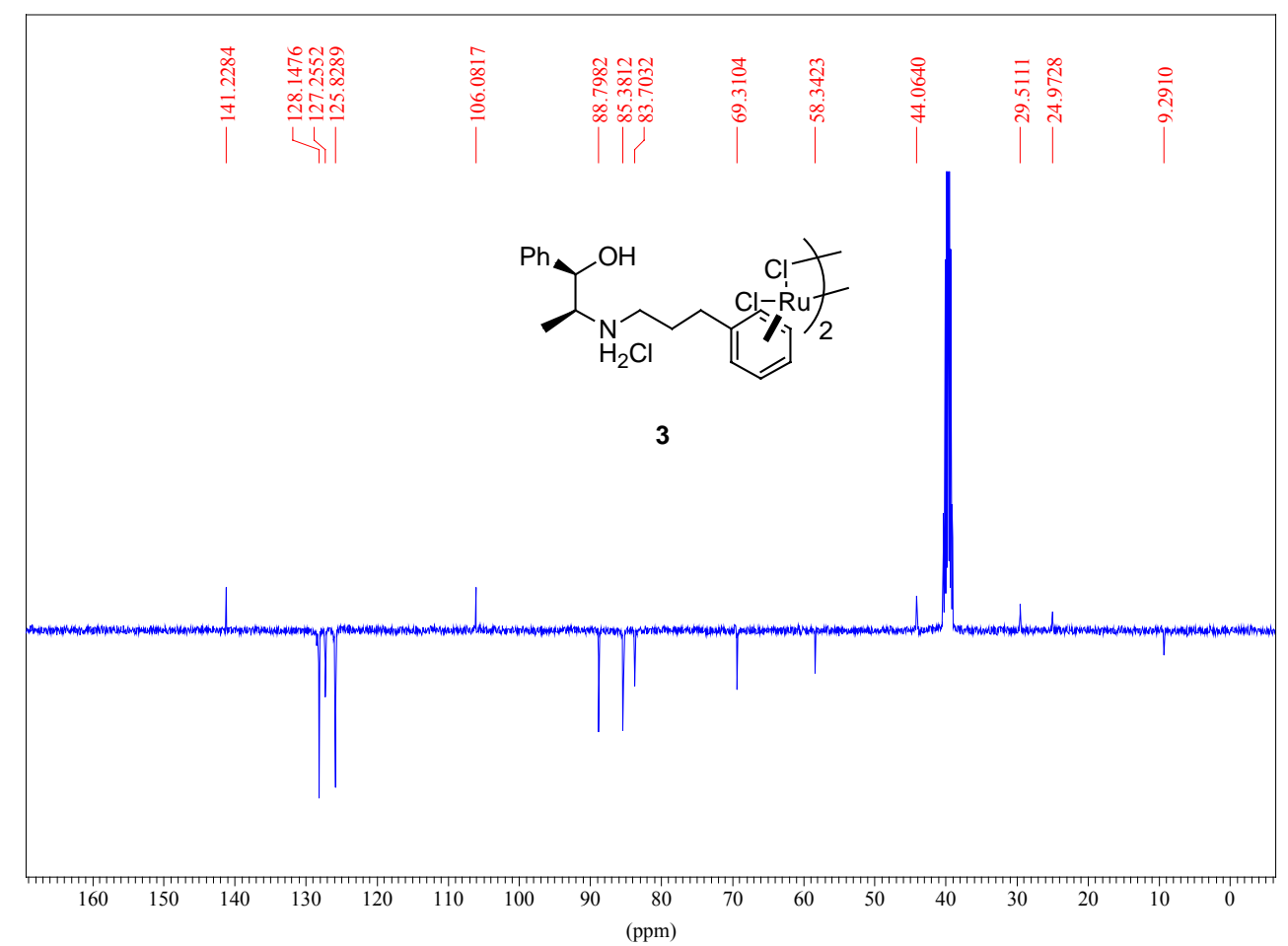

Parameters: C13pend. with DMSO $\quad \mathrm{SF}=100.53 \quad \mathrm{SW}=25125.63 \quad \mathrm{AQ}=1.30 \quad \mathrm{TD}=131072 \quad$ Units=ppm 


\section{M) Single X-ray data analysis data of 2}

\section{1) ORTEP plot of the molecular structure}

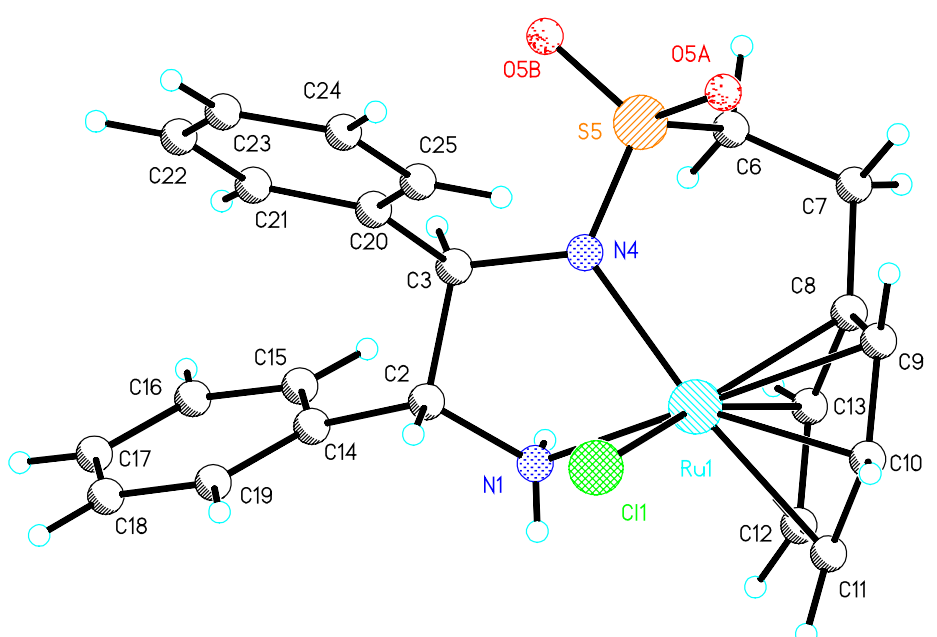

\section{2) Experimental Details}

The asymmetric unit contains one complex. There are two complexes in the unit cell. There are several traditional and no traditional hydrogen bonds in the structure. The amine hydrogens were found and are involved in hydrogen bonding to the $\mathrm{Cl}$ and sulphonamide oxygen of neighbouring molecules. There is no intermolecular $\mathrm{H}$ bonding.

Each complex forms three contacts with its neighbour. Receiving two $\mathrm{H}$ bonds via the sulphonamide oxygens and donating the third via the alpha carbon of the sulphonamide (see structure at the end of the document). This motif is replicated forming a helix that travels along the "b" axis of the crystal. Additionally, the chlorine forms further contacts in the "a" direction that link the helices together. These contacts are listed below

Specified hydrogen bonds (with esds except fixed and riding $\mathrm{H}$ )

\begin{tabular}{lllll} 
D-H & H...A & D...A & $<($ DHA $)$ & \\
\cline { 1 - 3 } $0.851(19)$ & $2.04(2)$ & $2.888(4)$ & $176(4)$ & \\
1.00 & 2.26 & $3.220(6)$ & 159.7 & C11-H1A...O5A \\
$0.85(4)$ & $2.67(4)$ & $3.504(4)$ & $168(4)$ & N1-H1B...C11 \\
1.00 & 2.50 & $3.402(4)$ & 149.7 & C13-H13A...C11 \\
0.99 & 2.91 & $3.712(5)$ & 138.4 & C6-H6B...C11
\end{tabular}

Least-squares planes (x,y,z in crystal coordinates) and deviations from them

(* indicates atom used to define plane)

* $0.0012(0.0031) \mathrm{C} 8$

* $0.0008(0.0032)$ C9

* $-0.0046(0.0028) \mathrm{C} 10$

* $0.0064(0.0033) \mathrm{C} 11$

* $-0.0045(0.0033) \mathrm{C} 12$

* $0.0007(0.0027) \mathrm{C} 13$

$-1.6596(0.0015) \mathrm{Ru} 1$

Deviation of least squares plane through the benzene ring (C8 to C13)from ruthenium ion (1.6596(0.0015) $\AA$ from ring).

\section{Crystal Data}

C22 H23 Cl N2 O2 Ru S, M= 516.00, 
Monoclinic, space group P2(1)

$\mathrm{a}=6.4552(8), \mathrm{b}=14.6777(18), \mathrm{c}=11.1916(14) \mathrm{A}$,

alpha $=90$ deg., beta $=102.127(3)$ deg., gamma $=90$ deg.,

$\mathrm{U}=1036.7(2) \mathrm{A}^{\wedge} 3$ (by least squares refinement on 4991 reflection positions),

$\mathrm{T}=180(2) \mathrm{K}$, lambda $=0.71073 \mathrm{~A}, \mathrm{Z}=2$,

$\mathrm{D}(\mathrm{cal})=1.653 \mathrm{Mg} / \mathrm{m}^{\wedge} 3, \mathrm{~F}(000)=524$.

$\mathrm{mu}(\mathrm{MoK}$-alpha $)=1.007 \mathrm{~mm}^{\wedge}-1$.

Crystal character: orange block.

Crystal dimensions $0.22 \times 0.16 \times 0.10 \mathrm{~mm}$,

\section{Data Collection and Processing}

Siemens SMART (Siemens, 1994) three-circle system with CCD area detector.

The crystal was held at 180(2)K with the Oxford Cryosystem Cryostream Cooler(Cosier \& Glazer, 1986).

Maximum theta was $29.15 \mathrm{deg}$.

The hkl ranges were $-8 / 8,-18 / 19,-6 / 15$.

6730 reflections measured, 4663 unique $[\mathrm{R}(\mathrm{int})=0.0257]$.

Absorption correction by Semi-empirical from equivalents;

minimum and maximum transmission factors: $0.68 ; 0.91$.

No crystal decay

\section{Structure Analysis and Refinement}

Systematic absences indicated either space group P2 (1) or P2 (1)/m.

The former was chosen because of the known chirality of the system and shown to be correct by successful refinement.

The structure was solved by direct methods using SHELXS (Sheldrick, 1990) (TREF) with additional light atoms found by

Fourier methods.

Hydrogen atoms were added at calculated positions and refined using a riding

Model except the hydrogens on the amine, which were found in a difference synthesis. Anisotropic displacement parameters were used for all non-H atoms;

$\mathrm{H}$-atoms were given isotropic displacement parameters equal to 1.2 (or 1.5 for the amine hydrogen atoms) times the equivalent isotropic displacement parameter of the atom to which the $\mathrm{H}$-atom is attached.

The chirality was checked by refinement of a delta-f" multiplier. Absolute structure parameter $\mathrm{x}=0.01(3)$.

Floating origin constraints were generated automatically.

The weighting scheme was calc $\mathrm{w}=1 /\left[\backslash \mathrm{s}^{\wedge} 2^{\wedge}\left(\mathrm{Fo}^{\wedge} 2^{\wedge}\right)+(0.0234 \mathrm{P})^{\wedge} 2^{\wedge}+0.0000 \mathrm{P}\right]$ where $\mathrm{P}=\left(\mathrm{Fo}^{\wedge} 2^{\wedge}+2 \mathrm{Fc}^{\wedge} 2^{\wedge}\right) / 3$.

Goodness-of-fit on $\mathrm{F}^{\wedge} 2$ was 0.945 ,

R1[for 3972 reflections with

$\mathrm{I}>2 \operatorname{sigma}(\mathrm{I})]=0.0326, \mathrm{wR} 2=0.0628$.

Data / restraints / parameters 4663/ 2/ 269.

Extinction coefficient $0.0163(11)$.

Largest difference Fourier peak and hole 0.412 and -0.763 e. $\mathrm{A}^{\wedge}-3$.

Refinement used SHELXTL (Sheldrick, 1997).

We thank EPSRC and Siemens Analytical Instruments for grants in support of the diffractometer.

Additional material available from the Cambridge Crystallographic Data Centre comprises $\mathrm{H}$-atom coordinates, thermal parameters and the remaining bond lengths and angles.

\section{References}

[ALCOCK, N.W. \& MARKS, P.J. (1994), J. Appl. Cryst. 27, 200-200.]

COSIER, J. \& GLAZER, A. M. (1986), J. Appl. Cryst. 19, 105-107.

SHELDRICK, G.M. (1990), Acta Cryst. A46, 467-473 
SHELDRICK, G.M. (1993), Acta Cryst. D49, 18-23

SHELDRICK, G.M.(1997), SHELXTL Ver. 5.1, Bruker Analytical X-ray Systems, Madison, Wis. USA.

SIEMENS (1994), SMART User's manual, Siemens Industrial Automation Inc, Madison, Wis. USA. 
3) Table 1. Atomic coordinates $\left(\times 10^{\wedge} 4\right)$ and equivalent isotropic displacement parameters $\left(\mathrm{A}^{\wedge} 2 \times 10^{\wedge} 3\right)$.

$\mathrm{U}(\mathrm{eq})$ is defined as one third of the trace of the orthogonalized Uij tensor.

\begin{tabular}{|c|c|c|c|c|}
\hline & $\mathrm{x}$ & $\mathrm{y}$ & $\mathrm{z}$ & $\mathrm{U}(\mathrm{eq})$ \\
\hline Ru1 & $5428.6(4)$ & $-4147.1(2)$ & $5593.2(2)$ & $18(1)$ \\
\hline $\mathrm{C} 11$ & $1773.3(14)$ & $-4684.5(7)$ & $4822.7(9)$ & $27(1)$ \\
\hline N1 & $6278(6)$ & $-5013(2)$ & $4267(3)$ & $17(1)$ \\
\hline $\mathrm{C} 2$ & $5014(6)$ & $-4805(3)$ & $3020(4)$ & $14(1)$ \\
\hline $\mathrm{C} 3$ & $4980(6)$ & $-3775(3)$ & $2830(4)$ & $16(1)$ \\
\hline $\mathrm{O} 5 \mathrm{~B}$ & $5792(5)$ & $-1936.4(18)$ & $2926(3)$ & $35(1)$ \\
\hline $\mathrm{O} 5 \mathrm{~A}$ & $4366(5)$ & $-1800(2)$ & $4796(3)$ & $38(1)$ \\
\hline N4 & $5011(5)$ & $-3329(2)$ & $4021(3)$ & $21(1)$ \\
\hline S5 & $5683.7(17)$ & $-2288.1(7)$ & $4122.8(9)$ & $26(1)$ \\
\hline C6 & $8294(7)$ & $-2210(4)$ & $5021(4)$ & $40(1)$ \\
\hline $\mathrm{C} 7$ & $8334(8)$ & $-2282(4)$ & $6381(4)$ & $44(1)$ \\
\hline $\mathrm{C} 8$ & $7457(7)$ & $-3160(3)$ & $6752(4)$ & $27(1)$ \\
\hline C9 & $5466(7)$ & $-3197(3)$ & $7110(4)$ & $28(1)$ \\
\hline $\mathrm{C} 10$ & $4653(6)$ & $-4027(4)$ & 7394(3) & $34(1)$ \\
\hline $\mathrm{C} 11$ & $5769(10)$ & $-4849(4)$ & $7351(4)$ & $39(1)$ \\
\hline $\mathrm{C} 12$ & $7721(9)$ & $-4812(4)$ & $6988(4)$ & $40(2)$ \\
\hline $\mathrm{C} 13$ & $8571(6)$ & $-3978(4)$ & $6698(4)$ & $32(1)$ \\
\hline $\mathrm{C} 14$ & $5826(6)$ & $-5320(2)$ & 2019(3) & $17(1)$ \\
\hline $\mathrm{C} 15$ & $7811(6)$ & $-5151(3)$ & $1787(4)$ & $24(1)$ \\
\hline $\mathrm{C} 16$ & $8461(7)$ & $-5552(3)$ & $818(4)$ & $30(1)$ \\
\hline $\mathrm{C} 17$ & $7125(7)$ & $-6148(3)$ & $58(4)$ & $31(1)$ \\
\hline $\mathrm{C} 18$ & $5172(7)$ & $-6333(3)$ & 292(4) & $35(1)$ \\
\hline C19 & $4515(6)$ & $-5923(3)$ & $1260(4)$ & $24(1)$ \\
\hline $\mathrm{C} 20$ & $3047(6)$ & $-3500(2)$ & $1858(3)$ & $18(1)$ \\
\hline $\mathrm{C} 21$ & $3037(6)$ & $-3645(3)$ & $635(4)$ & $28(1)$ \\
\hline $\mathrm{C} 22$ & $1239(7)$ & $-3423(3)$ & $-247(4)$ & $37(1)$ \\
\hline $\mathrm{C} 23$ & $-508(7)$ & $-3052(3)$ & $74(4)$ & $33(1)$ \\
\hline $\mathrm{C} 24$ & $-470(7)$ & $-2879(3)$ & $1286(4)$ & $29(1)$ \\
\hline $\mathrm{C} 25$ & $1284(6)$ & $-3109(3)$ & $2176(4)$ & $24(1)$ \\
\hline
\end{tabular}


4) Table 2. Bond lengths [A] and angles [deg].

\begin{tabular}{|c|c|}
\hline Ru1-N4 & $2.100(3)$ \\
\hline Ru1-N1 & $2.112(3)$ \\
\hline $\mathrm{Ru} 1-\mathrm{C} 12$ & $2.147(5)$ \\
\hline Ru1-C13 & $2.156(4)$ \\
\hline $\mathrm{Ru} 1-\mathrm{C} 10$ & $2.183(4)$ \\
\hline Ru1-C8 & $2.187(4)$ \\
\hline Ru1-C11 & $2.191(5)$ \\
\hline Ru1-C9 & $2.193(5)$ \\
\hline Ru1-Cl1 & $2.4655(10)$ \\
\hline $\mathrm{N} 1-\mathrm{C} 2$ & $1.492(5)$ \\
\hline N1-H1A & $0.851(19)$ \\
\hline N1-H1B & $0.85(4)$ \\
\hline $\mathrm{C} 2-\mathrm{C} 3$ & $1.526(5)$ \\
\hline C2-C14 & $1.532(5)$ \\
\hline $\mathrm{C} 2-\mathrm{H} 2 \mathrm{~A}$ & 1.0000 \\
\hline $\mathrm{C} 3-\mathrm{N} 4$ & $1.482(5)$ \\
\hline C3-C20 & $1.528(5)$ \\
\hline $\mathrm{C} 3-\mathrm{H} 3 \mathrm{~A}$ & 1.0000 \\
\hline O5B-S5 & $1.450(3)$ \\
\hline O5A-S5 & $1.440(3)$ \\
\hline N4-S5 & $1.586(3)$ \\
\hline S5-C6 & $1.775(4)$ \\
\hline C6-C7 & $1.520(6)$ \\
\hline C6-H6A & 0.9900 \\
\hline C6-H6B & 0.9900 \\
\hline $\mathrm{C} 7-\mathrm{C} 8$ & $1.501(7)$ \\
\hline C7-H7A & 0.9900 \\
\hline C7-H7B & 0.9900 \\
\hline C8-C13 & $1.407(6)$ \\
\hline $\mathrm{C} 8-\mathrm{C} 9$ & $1.425(6)$ \\
\hline C9-C10 & $1.389(8)$ \\
\hline C9-H9A & 1.0000 \\
\hline $\mathrm{C} 10-\mathrm{C} 11$ & $1.412(8)$ \\
\hline C10-H10A & 1.0000 \\
\hline C11-C12 & $1.404(8)$ \\
\hline C11-H11A & 1.0000 \\
\hline C12-C13 & $1.407(7)$ \\
\hline C12-H12A & 1.0000 \\
\hline C13-H13A & 1.0000 \\
\hline C14-C15 & $1.382(5)$ \\
\hline C14-C19 & $1.385(5)$ \\
\hline $\mathrm{C} 15-\mathrm{C} 16$ & $1.375(6)$ \\
\hline C15-H15A & 0.9500 \\
\hline $\mathrm{C} 16-\mathrm{C} 17$ & $1.387(6)$ \\
\hline C16-H16A & 0.9500 \\
\hline C17-C18 & $1.367(6)$ \\
\hline C17-H17A & 0.9500 \\
\hline C18-C19 & $1.382(6)$ \\
\hline C18-H18A & 0.9500 \\
\hline C19-H19A & 0.9500 \\
\hline C20-C21 & $1.384(5)$ \\
\hline $\mathrm{C} 20-\mathrm{C} 25$ & $1.386(5)$ \\
\hline $\mathrm{C} 21-\mathrm{C} 22$ & $1.395(6)$ \\
\hline C21-H21A & 0.9500 \\
\hline $\mathrm{C} 22-\mathrm{C} 23$ & $1.367(6)$ \\
\hline $\mathrm{C} 22-\mathrm{H} 22 \mathrm{~A}$ & 0.9500 \\
\hline C23-C24 & $1.374(6)$ \\
\hline $\mathrm{C} 23-\mathrm{H} 23 \mathrm{~A}$ & 0.9500 \\
\hline
\end{tabular}




\begin{tabular}{|c|c|}
\hline $\begin{array}{l}\mathrm{C} 24-\mathrm{C} 25 \\
\mathrm{C} 24-\mathrm{H} 24 \mathrm{~A} \\
\mathrm{C} 25-\mathrm{H} 25 \mathrm{~A}\end{array}$ & $\begin{array}{l}1.384(5) \\
0.9500 \\
0.9500\end{array}$ \\
\hline N4-Ru1-N1 & $6.22(12)$ \\
\hline N4-Ru1-C12 & $144.8(2)$ \\
\hline N1-Ru1-C12 & $89.81(17)$ \\
\hline N4-Ru1-C13 & $110.73(16)$ \\
\hline N1-Ru1-C13 & $96.91(15)$ \\
\hline C12-Ru1-C13 & $38.17(19)$ \\
\hline N4-Ru1-C10 & $135.48(19)$ \\
\hline $\mathrm{N} 1-\mathrm{Ru} 1-\mathrm{C} 10$ & $147.3(2)$ \\
\hline C12-Ru1-C10 & $67.8(2)$ \\
\hline C13-Ru1-C10 & $80.34(15)$ \\
\hline N4-Ru1-C8 & $94.22(15)$ \\
\hline N1-Ru1-C8 & $127.16(15)$ \\
\hline C12-Ru1-C8 & $68.6(2)$ \\
\hline C13-Ru1-C8 & $37.80(17)$ \\
\hline C10-Ru1-C8 & $67.90(18)$ \\
\hline N4-Ru1-C11 & $172.88(18)$ \\
\hline N1-Ru1-C11 & $110.88(18)$ \\
\hline C12-Ru1-C11 & $37.8(2)$ \\
\hline C13-Ru1-C11 & $68.57(19)$ \\
\hline C10-Ru1-C11 & $37.7(2)$ \\
\hline C8-Ru1-C11 & $81.04(18)$ \\
\hline N4-Ru1-C9 & $105.28(17)$ \\
\hline N1-Ru1-C9 & $164.64(14)$ \\
\hline C12-Ru1-C9 & $80.34(17)$ \\
\hline C13-Ru1-C9 & $68.10(16)$ \\
\hline C10-Ru1-C9 & $37.0(2)$ \\
\hline C8-Ru1-C9 & $37.97(17)$ \\
\hline C11-Ru1-C9 & $67.74(19)$ \\
\hline N4-Ru1-Cl1 & $86.85(9)$ \\
\hline N1-Ru1-C11 & $86.12(10)$ \\
\hline C12-Ru1-Cl1 & $124.87(16)$ \\
\hline C13-Ru1-C11 & $162.40(13)$ \\
\hline C10-Ru1-Cl1 & $87.73(11)$ \\
\hline C8-Ru1-C11 & $146.02(13)$ \\
\hline C11-Ru1-Cl1 & $94.11(15)$ \\
\hline C9-Ru1-Cl1 & $109.17(12)$ \\
\hline C2-N1-Ru1 & $111.3(2)$ \\
\hline C2-N1-H1A & $117(3)$ \\
\hline Ru1-N1-H1A & $104(3)$ \\
\hline C2-N1-H1B & $109(3)$ \\
\hline Ru1-N1-H1B & $104(3)$ \\
\hline H1A-N1-H1B & $111(4)$ \\
\hline N1-C2-C3 & $108.8(3)$ \\
\hline $\mathrm{N} 1-\mathrm{C} 2-\mathrm{C} 14$ & $112.3(3)$ \\
\hline C3-C2-C14 & $112.5(4)$ \\
\hline $\mathrm{N} 1-\mathrm{C} 2-\mathrm{H} 2 \mathrm{~A}$ & 107.7 \\
\hline $\mathrm{C} 3-\mathrm{C} 2-\mathrm{H} 2 \mathrm{~A}$ & 107.7 \\
\hline $\mathrm{C} 14-\mathrm{C} 2-\mathrm{H} 2 \mathrm{~A}$ & 107.7 \\
\hline $\mathrm{N} 4-\mathrm{C} 3-\mathrm{C} 2$ & $108.4(4)$ \\
\hline N4-C3-C20 & 112.2(3) \\
\hline $\mathrm{C} 2-\mathrm{C} 3-\mathrm{C} 20$ & $110.3(3)$ \\
\hline $\mathrm{N} 4-\mathrm{C} 3-\mathrm{H} 3 \mathrm{~A}$ & 108.6 \\
\hline $\mathrm{C} 2-\mathrm{C} 3-\mathrm{H} 3 \mathrm{~A}$ & 108.6 \\
\hline $\mathrm{C} 20-\mathrm{C} 3-\mathrm{H} 3 \mathrm{~A}$ & 108.6 \\
\hline C3-N4-S5 & $116.2(3)$ \\
\hline C3-N4-Ru1 & $118.4(2)$ \\
\hline
\end{tabular}




\begin{tabular}{|c|c|}
\hline S5-N4-Ru1 & $120.39(19)$ \\
\hline O5A-S5-O5B & $117.02(19)$ \\
\hline O5A-S5-N4 & $109.50(19)$ \\
\hline O5B-S5-N4 & $110.02(17)$ \\
\hline O5A-S5-C6 & $105.4(2)$ \\
\hline O5B-S5-C6 & 106.01(19) \\
\hline N4-S5-C6 & $108.4(2)$ \\
\hline C7-C6-S5 & $112.1(3)$ \\
\hline C7-C6-H6A & 109.2 \\
\hline S5-C6-H6A & 109.2 \\
\hline C7-C6-H6B & 109.2 \\
\hline S5-C6-H6B & 109.2 \\
\hline H6A-C6-H6B & 107.9 \\
\hline C8-C7-C6 & $114.1(4)$ \\
\hline C8-C7-H7A & 108.7 \\
\hline C6-C7-H7A & 108.7 \\
\hline C8-C7-H7B & 108.7 \\
\hline C6-C7-H7B & 108.7 \\
\hline H7A-C7-H7B & 107.6 \\
\hline C13-C8-C9 & $118.6(4)$ \\
\hline $\mathrm{C} 13-\mathrm{C} 8-\mathrm{C} 7$ & $119.6(4)$ \\
\hline C9-C8-C7 & $121.7(5)$ \\
\hline C13-C8-Ru1 & $69.9(2)$ \\
\hline C9-C8-Ru1 & $71.3(3)$ \\
\hline C7-C8-Ru1 & $128.2(3)$ \\
\hline $\mathrm{C} 10-\mathrm{C} 9-\mathrm{C} 8$ & $120.3(4)$ \\
\hline C10-C9-Ru1 & $71.1(3)$ \\
\hline C8-C9-Ru1 & $70.8(3)$ \\
\hline C10-C9-H9A & 119.2 \\
\hline C8-C9-H9A & 119.2 \\
\hline Ru1-C9-H9A & 119.2 \\
\hline C9-C10-C11 & $121.5(4)$ \\
\hline C9-C10-Ru1 & $71.9(3)$ \\
\hline C11-C10-Ru1 & $71.5(3)$ \\
\hline C9-C10-H10A & 118.6 \\
\hline C11-C10-H10A & 118.6 \\
\hline Ru1-C10-H10A & 118.6 \\
\hline C12-C11-C10 & $118.2(5)$ \\
\hline C12-C11-Ru1 & $69.4(3)$ \\
\hline C10-C11-Ru1 & $70.9(3)$ \\
\hline C12-C11-H11A & 120.3 \\
\hline C10-C11-H11A & 120.3 \\
\hline Ru1-C11-H11A & 120.3 \\
\hline C11-C12-C13 & $121.2(5)$ \\
\hline C11-C12-Ru1 & $72.8(3)$ \\
\hline C13-C12-Ru1 & $71.3(3)$ \\
\hline $\mathrm{C} 11-\mathrm{C} 12-\mathrm{H} 12 \mathrm{~A}$ & 118.9 \\
\hline C13-C12-H12A & 118.9 \\
\hline $\mathrm{Ru} 1-\mathrm{C} 12-\mathrm{H} 12 \mathrm{~A}$ & 118.9 \\
\hline C8-C13-C12 & $120.3(4)$ \\
\hline C8-C13-Ru1 & $72.3(2)$ \\
\hline C12-C13-Ru1 & $70.5(3)$ \\
\hline C8-C13-H13A & 119.3 \\
\hline C12-C13-H13A & 119.3 \\
\hline Ru1-C13-H13A & 119.3 \\
\hline C15-C14-C19 & 118.1(3) \\
\hline C15-C14-C2 & $121.5(3)$ \\
\hline C19-C14-C2 & $120.3(3)$ \\
\hline C16-C15-C14 & 121.3(4) \\
\hline C16-C15-H15A & 119.3 \\
\hline C14-C15-H15A & 119.3 \\
\hline C15-C16-C17 & $120.0(4)$ \\
\hline
\end{tabular}




\begin{tabular}{ll} 
C15-C16-H16A & 120.0 \\
C17-C16-H16A & 120.0 \\
C18-C17-C16 & $119.1(4)$ \\
C18-C17-H17A & 120.5 \\
C16-C17-H17A & 120.5 \\
C17-C18-C19 & $120.8(4)$ \\
C17-C18-H18A & 119.6 \\
C19-C18-H18A & 119.6 \\
C18-C19-C14 & $120.6(4)$ \\
C18-C19-H19A & 119.7 \\
C14-C19-H19A & 119.7 \\
C21-C20-C25 & $119.0(3)$ \\
C21-C20-C3 & $119.8(3)$ \\
C25-C20-C3 & $121.2(3)$ \\
C20-C21-C22 & $119.6(4)$ \\
C20-C21-H21A & 120.2 \\
C22-C21-H21A & 120.2 \\
C23-C22-C21 & $121.1(4)$ \\
C23-C22-H22A & 119.4 \\
C21-C22-H22A & 119.4 \\
C22-C23-C24 & $119.2(4)$ \\
C22-C23-H23A & 120.4 \\
C24-C23-H23A & 120.4 \\
C23-C24-C25 & $120.5(4)$ \\
C23-C24-H24A & 119.7 \\
C25-C24-H24A & 119.7 \\
C24-C25-C20 & $120.5(4)$ \\
C24-C25-H25A & 119.7 \\
C20-C25-H25A & 119.7 \\
& \\
\hline
\end{tabular}

Symmetry transformations used to generate equivalent atoms. 
5) Table 3. Anisotropic displacement parameters $\left(A^{\wedge} 2 \times 10^{\wedge} 3\right)$.

The anisotropic displacement factor exponent takes the form: $-2 \mathrm{pi}^{\wedge} 2\left[\mathrm{~h}^{\wedge} 2 \mathrm{a}^{* \wedge} 2 \mathrm{U} 11+\ldots+2 \mathrm{~h} \mathrm{k} \mathrm{a} \mathrm{b}^{*} \mathrm{U} 12\right.$ ]

\begin{tabular}{|c|c|c|c|c|c|c|}
\hline & U11 & U22 & U33 & U23 & U13 & U12 \\
\hline Ru1 & $19(1)$ & $21(1)$ & 11(1) & $-3(1)$ & $-1(1)$ & $3(1)$ \\
\hline $\mathrm{Cl1}$ & $19(1)$ & $39(1)$ & $22(1)$ & $-4(1)$ & $4(1)$ & $0(1)$ \\
\hline N1 & $16(2)$ & $18(2)$ & $14(2)$ & $1(1)$ & $0(1)$ & $-3(1)$ \\
\hline $\mathrm{C} 2$ & $16(2)$ & $11(2)$ & $15(2)$ & $0(2)$ & $0(2)$ & $3(2)$ \\
\hline $\mathrm{C} 3$ & $19(2)$ & $15(2)$ & $13(2)$ & $-4(2)$ & $1(2)$ & $2(2)$ \\
\hline O5B & $56(2)$ & $21(2)$ & $25(2)$ & $3(1)$ & $4(2)$ & $-7(1)$ \\
\hline O5A & $57(2)$ & $19(2)$ & $38(2)$ & $-15(1)$ & $12(2)$ & $5(1)$ \\
\hline N4 & $31(2)$ & $15(2)$ & $17(2)$ & $-2(1)$ & $1(2)$ & $4(2)$ \\
\hline S5 & $38(1)$ & $16(1)$ & $22(1)$ & $-5(1)$ & $3(1)$ & $-1(1)$ \\
\hline C6 & $43(3)$ & $41(3)$ & $32(3)$ & $-3(2)$ & $2(2)$ & $-21(2)$ \\
\hline $\mathrm{C} 7$ & $51(3)$ & $51(3)$ & $26(2)$ & $-3(2)$ & $-2(2)$ & $-22(2)$ \\
\hline $\mathrm{C} 8$ & $28(2)$ & $38(3)$ & $14(2)$ & $-9(2)$ & $-2(2)$ & $-7(2)$ \\
\hline C9 & $22(2)$ & $40(3)$ & $20(2)$ & $-9(2)$ & $-2(2)$ & $13(2)$ \\
\hline $\mathrm{C} 10$ & $31(2)$ & $57(4)$ & $15(2)$ & $-8(3)$ & $5(2)$ & $-4(3)$ \\
\hline C11 & $64(4)$ & $36(3)$ & $10(2)$ & $5(2)$ & $-8(2)$ & $-11(3)$ \\
\hline C12 & $56(4)$ & $37(3)$ & $17(2)$ & $-9(2)$ & $-16(2)$ & $23(3)$ \\
\hline $\mathrm{C} 13$ & $26(2)$ & $49(4)$ & $15(2)$ & $-14(2)$ & $-6(2)$ & $7(2)$ \\
\hline C14 & $27(2)$ & $14(2)$ & $9(2)$ & $0(2)$ & $-1(2)$ & $-1(2)$ \\
\hline $\mathrm{C} 15$ & $20(2)$ & $26(2)$ & $23(2)$ & $-5(2)$ & $1(2)$ & $1(2)$ \\
\hline C16 & $28(2)$ & $31(2)$ & $34(3)$ & $3(2)$ & $13(2)$ & $9(2)$ \\
\hline $\mathrm{C} 17$ & $52(3)$ & $27(2)$ & $18(2)$ & $-2(2)$ & $14(2)$ & $12(2)$ \\
\hline C18 & $45(3)$ & $35(3)$ & $23(2)$ & $-16(2)$ & $3(2)$ & $-6(2)$ \\
\hline C19 & $27(2)$ & $21(2)$ & $23(2)$ & $-7(2)$ & $5(2)$ & $-8(2)$ \\
\hline $\mathrm{C} 20$ & $20(2)$ & $18(2)$ & $15(2)$ & $1(2)$ & $-2(2)$ & $-1(2)$ \\
\hline $\mathrm{C} 21$ & $33(2)$ & $29(2)$ & $20(2)$ & $-1(2)$ & $4(2)$ & $6(2)$ \\
\hline $\mathrm{C} 22$ & $51(3)$ & $38(3)$ & $17(2)$ & $1(2)$ & $-4(2)$ & $4(2)$ \\
\hline $\mathrm{C} 23$ & $26(2)$ & $39(3)$ & $28(2)$ & $2(2)$ & $-8(2)$ & $4(2)$ \\
\hline $\mathrm{C} 24$ & $24(2)$ & $31(2)$ & $29(2)$ & $15(2)$ & $2(2)$ & $4(2)$ \\
\hline $\mathrm{C} 25$ & $27(2)$ & $24(2)$ & $19(2)$ & $5(2)$ & $3(2)$ & $3(2)$ \\
\hline
\end{tabular}


6) Table 4.Hydrogen coordinates $\left(\times 10^{\wedge} 4\right)$ and isotropic displacement parameters $\left(A^{\wedge} 2 \times 10^{\wedge} 3\right)$.

\begin{tabular}{lllll}
\hline & & & & \\
& & & & \\
& & & & \\
H1A & $6150(60)$ & $-5546(17)$ & $4540(40)$ & 25 \\
H1B & $7580(60)$ & $-4880(30)$ & $4290(40)$ & 25 \\
H2A & 3525 & -5007 & 2990 & 17 \\
H3A & 6288 & -3594 & 2543 & 19 \\
H6A & 9171 & -2704 & 4783 & 47 \\
H6B & 8925 & -1621 & 4854 & 47 \\
H7A & 9816 & -2216 & 6838 & 53 \\
H7B & 7508 & -1770 & 6620 & 53 \\
H9A & 4519 & -2651 & 6978 & 34 \\
H10A & 3131 & -4058 & 7456 & 41 \\
H11A & 5053 & -5448 & 7402 & 47 \\
H12A & 8381 & -5392 & 6785 & 48 \\
H13A & 9815 & -3981 & 6295 & 38 \\
H15A & 8745 & -4750 & 2308 & 28 \\
H16A & 9825 & -5421 & 669 & 36 \\
H17A & 7561 & -6425 & -617 & 38 \\
H18A & 4257 & -6747 & -217 & 42 \\
H19A & 3150 & -6056 & 1408 & 29 \\
H21A & 4248 & -3895 & 397 & 33 \\
H22A & 1228 & -3532 & -1085 & 44 \\
H23A & -1735 & -2915 & -533 & 40 \\
H24A & -1656 & -2598 & 1513 & 34 \\
H25A & 1279 & -2999 & 3012 & 28 \\
& & & & \\
\hline
\end{tabular}

Structure showing the interactions between 2 molecules

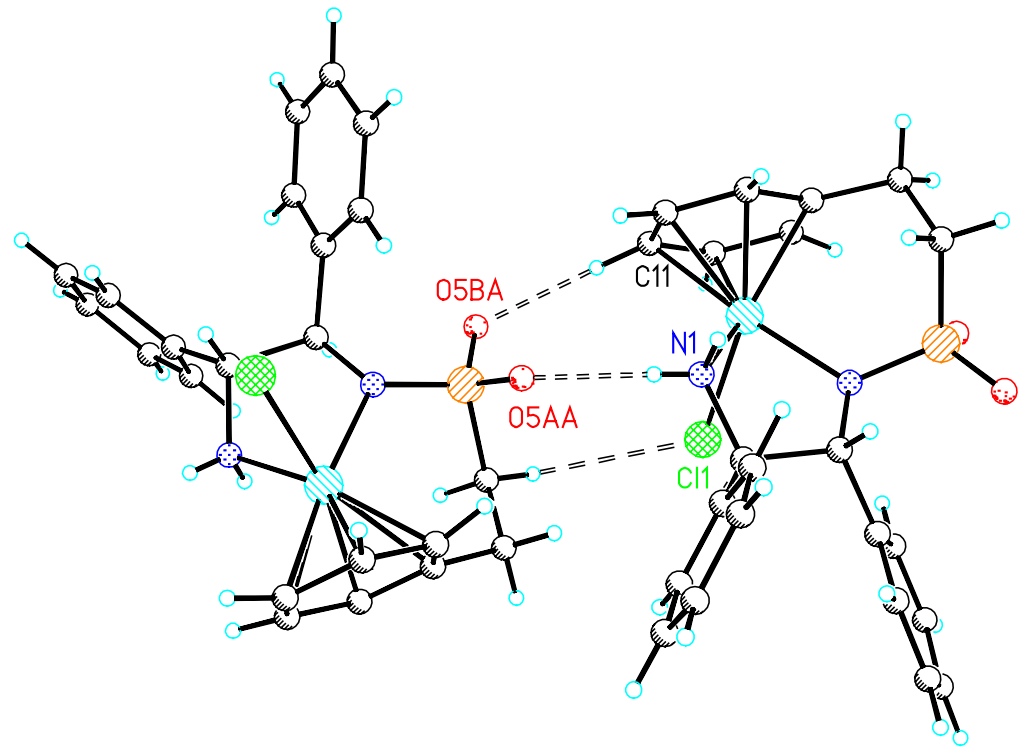

Interaction between two molecules with amine sulphonamide $\mathrm{H}$ bonding $(\mathrm{NH} 1 \mathrm{a} . . . \mathrm{O} 5 \mathrm{a}$ ) and $\mathrm{CH}$ hydrogen bonds between the sulphonamide and aromatic $\mathrm{H}(\mathrm{C} 11-\mathrm{H} 11 \mathrm{a} . . \mathrm{O} 5 \mathrm{a})$ and the chlorine and an alpha proton of the sulphonamide (C6-H6a ... C11). 


\section{N) References}

${ }^{1}$ Procedure adapted from Block, E.; Wall, A. J. Org. Chem. 1987, 52, 809.

${ }^{2}$ Birch Reduction: Birch, A. J. J. Chem. Soc 1950, 1551.

${ }^{3}$ Procedure adapted from Meuzelaar, G.J.; van Vliet M.C.A.; Maat, L.; Sheldon, R.A. Eur.J.Org. Chem. 1999, 2315.

${ }^{4}$ Procedure adapted from Miyaki, Y.; Onishi, T.; Kurosawa, H. Inorg. Chim. Acta 2000, 300-302, 369; Bennett, M. A., `Smith, A. K. J. Chem. Soc., Dalton Trans. 1974, 233.

${ }^{5}$ Procedure adapted from Haack, K. J.; Hashiguchi, S.; Fujii, A.; Ikariya, T.; Noyori, R. Angew. Chem. Int. Ed. 1997, $36,285$.

${ }^{6}$ Hayashi, T.; Matsumoto, Y.; Ito, Y. Tetrahedron: Asymmetry 1991, 2, 601.

${ }^{7}$ Fujii, A.; Hashiguchi, S.; Uematsu, N.; Ikariya, T.; Noyori, R. J. Am. Chem. Soc. 1996, 118, 2521.

${ }^{8}$ Palmer, M.; Walsgrove, T.; Wills, M. J. Org. Chem. 1997, 62, 5226.

${ }^{9}$ Hayashi,T.; Matsumoto, Y.; Ito, Y. J. Am. Chem. Soc. 1989, 111, 3426.

${ }^{10}$ Carter, M. B.; Schiøtt, B.; Gutiérrez, A.; Buchwald, S. L. J. Am. Chem. Soc. 1994, 116, 11667.

${ }^{11}$ Yadav, J. S.; Nanda, S.; Thirupathi Reddy, P.; Bhaskar Rao, A. J.Org.Chem. 2002, 67, 3900. 